\title{
Akkumulation von Metallen und Stickstoff in Moosen in Nordrhein-Westfalen 1990 - 2005 (Accumulation of metals and nitrogen in mosses in North Rhine-Westfalia 1990-2005)
}

\author{
Winfried Schröder ${ }^{1 *}$, Roland Pesch ${ }^{1 *}$, Marcel Holy ${ }^{1 *}$, Lutz Genßler ${ }^{2}$, Harry Harmens ${ }^{3}$ and llia llyin ${ }^{4}$
}

\begin{abstract}
Zusammenfassung
Hintergrund: Umweltbeobachtung ist zentraler Bestandteil internationaler Nachhaltigkeitsstrategien. Die langfristige Beobachtung der Anreicherung von Metallen in terrestrischen Ökosystemen erfolgte Zwischen 1990 und 2005 alle fünf Jahre europaweit in den europäischen Heavy Metals in Mosses Surveys. Seit 2005 wird auch die Stickstoffanreicherung bestimmt. Deutschland nahm an den Moosmonitoring-Kampagnen $1990-2005$ teil. Der vorliegende Artikel ist Teil einer Serie, die Trends im Bundesgebiet und einzelnen Bundesländern beleuchtet. Gegenstand dieser Untersuchung ist die Kartierung der zeitlichen Entwicklung der Metallanreicherung in Nordrhein-Westfalen seit 1990, die Stickstoffanreicherung 2005, die räumliche Varianz der Metall-Bioakkumulation in Abhängigkeit von Eigenschaften der Moosbeprobungsstellen und ihrer Umgebung sowie erstmals auch die Verknüpfung der Stoffgehalte in den Moosen mit denen der flächendeckend modellierten Gesamtdeposition von Cadmium (Cd).
\end{abstract}

Methoden: In Nordrhein-Westfalen wurde die Bioakkumulation am häufigsten in Scleropodium purum bestimmt, gefolgt von Pleurozium schreberi und Hypnum cupressiforme. Die in diesen Moosen chemisch bestimmten Stoffe wurden mit den topografischen und ökologischen Beschreibungen der bis zu 88 Beprobungsorte und mit quantitativen Angaben über die Landnutzung in ihrer Umgebung in dem WebGIS MossMet zusammengeführt und ausgewertet: Aus den standort- und metallspezifischen Messdaten sowie den daraus geostatistisch berechneten Flächendaten über die Metallakkumulation wurde ein zusammenfassender Multi-Metall-Index $\left(\mathrm{MMI}_{1990-2005}\right)$ für Arsen (As), Cd, Chrom (Cr), Kupfer (Cu), Eisen (Fe), Nickel (Ni), Blei (Pb), Titan (Ti), Vanadium (V) und Zink (Zn) berechnet und kartiert. Die Zusammenhänge zwischen den Schwermetall-Akkumulationen, Standortcharakteristika und Landnutzung wurden korrelations- und kontingenzanalytisch sowie am Beispiel von Cu multivariat-statistisch mit Classification and Regression Trees (Cart) quantifiziert. Die Cd-Gehalte in den Moosen wurden mit denen der im European Monitoring and Evaluation Programme (EMEP) europaweit flächendeckend modellierten Gesamtdepositionsdaten für Cd verknüpft und korrelationsstatistisch ausgewertet.

Ergebnisse und Diskussion: Während von 1990 bis 2005 bis auf Zn alle Metallgehalte in den Moosen sanken, stiegen von 2000 bis 2005 die Konzentrationen von As, Cr, Cu, Ni, Sb und Zn an, bei Cr und Zn statistisch signifikant. Eine Zunahme des $\mathrm{MMI}_{1990-2005}$ von 2000 nach 2005 erwies sich als statistisch nicht signifikant. Die Stickstoffgehalte $(\mathrm{N})$ betragen zwischen 1.08 und 2,29\%. Sie sind positiv mit dem Agrarflächenanteil im Umkreis der (Continued on next page)

\footnotetext{
* Correspondence: wschroeder@iuw.uni-vechta.de; rpesch@iuw.uni-vechta.de;

mholy@iuw.uni-vechta.de

${ }^{1}$ Lehrstuhl für Landschaftsökologie, Universität Vechta, Postfach 1553, 49364,

Vechta, Germany

Full list of author information is available at the end of the article
} 
(Continued from previous page)

Beprobungsstellen und der Bestandeshöhe sowie negativ mit Waldflächenanteil, Entfernung zu Bäumen, Höhe über NN und Niederschlag korreliert $\left(0.32 \leq r_{\text {Spearman }} \geq 0.49, p<0.01\right)$. Die Korrelationen zwischen Metallgehalten in den Moosen und der Landnutzung im Umkreis der Beprobungsorte rangieren zwischen $r_{S}=0.21$ und $r_{S}=0.54(0.01<p$ $<0.05)$. Moosart und -bewuchsform sind mit den Stoffkonzentrationen ähnlich stark assoziiert (Cramér's V-Werte zwischen 0.27 und 0.56). Von den Standortmerkmalen weisen vor allem die Variablen Waldflächenanteil (insbesondere bei $\mathrm{Cd}, \mathrm{Cu}, \mathrm{Pb}, \mathrm{Zn}, \mathrm{N}$ ), Flächenanteil urbaner Landnutzung (bei As, Cd, Cr, Cu, Fe, Ni, Ti, Zn), Niederschlagssumme im Akkumulationszeitraum (bei Cd, Ni, Pb, V, N), orografische Höhe (bei As, Cd, Cr, Cu, Fe, Ni, Ti, Zn, N) und Entfernung der Moos-Entnahmestelle von Straßen (bei Cr, Fe, Ni, Ti), Baumkronen oder Sträuchern (bei As, Cd, Cr, Cu, Fe, Ni, Zn) für die meisten Elemente signifikante Korrelationen zur Metallanreicherung auf. In der multivariat-statistischen Analyse mit CART werden der urbane Flächenanteil im Umkreis von 5 km um die Moossammelstelle sowie die dortige Geländehöhe und die Entfernung der Moossammelstelle von der Baumkrone als wichtigste Einflussgrößen für die Cu-Gehalte in den Moosen 2005 ermittelt. Die Cd-Gesamtdeposition (EMEP) und die Cd-Konzentrationen in Moosen Nordrhein-Westfalens sind positiv korreliert $\left(0.57 \leq r_{S} \geq 0.71, p<0.01\right)$. Anders als etwa in Baden-Württemberg stiegen die Metallanreicherungen in Moosen Nordrhein-Westfalens von 2000 bis 2005 an, Cr und Zn statistisch signifikant. Für Cd konnte in einer landesweit flächendeckenden GIS-gestützten Korrelationsanalyse gezeigt werden, dass die in den Moosen gemessenen Anreicherungen mit der modellierten Gesamtdeposition (EMEP) positiv verknüpft sind. Damit wurden punktuelle Korrelationen zwischen Depositions- und Mooskonzentrationen räumlich validiert. Im Vergleich zu zeitlich höher aufgelösten Depositionsmessungen erfasst das Moos-Monitoring europaweit mit mindestens einer Moosbeprobungsstelle pro $1000 \mathrm{~km}^{2}$ ein breites Stoffspektrum, das auch selten gemessene Stoffe mit humantoxikologischer Bedeutung (z. B. As, Al, Hg, Sb, V) umfasst. Damit bildet das Moos-Monitoring ein wichtiges Bindeglied zwischen der technischen Erfassung von Stoffeinträgen durch Deposition und der Anreicherung dieser Stoffe in biologischem Material. Die Untersuchung zeigt, dass die Stoffanreicherung in biologischem Material nicht nur von den Depositionen, sondern auch von topographischen und ökologischen Merkmalen der Messstellen und der Landnutzung ihrer Umgebung abhängt.

Schlussfolgerungen: Das Moos-Monitoring liefert wesentliche Beiträge zum Schwermetall- und zum Multi-Komponenten-Protokoll der CLRTAP. Es weist flächendeckend nach, wie sich Luftreinhaltepolitik auf die Anreicherung von atmosphärischen Stoffeinträgen in Schutzgütern wie der Vegetation auswirkt. Von besonderer umweltpolitischer Bedeutung ist, dass in keinem anderen Messprogramm räumlich so verdichtet Daten über ein breites, ökotoxikologisch und humanmedizinisch bedeutsames Stoffspektrum erhoben werden. Die räumliche Auflösung von Umweltinformationen ist ein wesentliches Kriterium für ihre Nutzbarkeit im Vollzug umweltpolitischer Maßnahmen auf Bundes- und Länderebene.

Das Moos-Monitoring sollte im bisherigen Umfang langfristig fortgesetzt werden. Es liefert als einziges Messnetz in Europa räumlich hinreichend differenzierte, flächendeckende Informationen über die Metall- und Stickstoffexposition naturnaher und agrarisch genutzter Ökosysteme, die auch für einzelne Staaten und deren administrative Untergliederungen räumlich aussagekräftig sind. Die in anderen Untersuchungen jüngst belegten europaweiten Korrelationen zwischen Stoffanreicherungen in Moosen und EMEP-Depositionsdaten wurden in anderen Arbeiten dazu genutzt, die Kartierung der Metall- und Stickstoffdepositionen räumlich höher aufzulösen.

Schlagwörter: Bioakkumulation, CART, EMEP-Cd-Deposition, Geostatistik, ICP Vegetation, Schwermetalle, Stickstoff

\section{Abstract}

Every five years since 1990, the European moss surveys provide data on concentrations of heavy metals and since 2005 on nitrogen (N) in mosses. Germany participated in the monitoring campaigns 1990 - 2005. As part of a series reporting the trends for Germany and single federal states, this article is on North Rhine-Westphalia showing that the metal concentrations decreased from 1990 to 2000 for all elements but Zn. From 2000 to 2005 an increase can be stated for As, Cr, Cu, Ni, Sb, Zn and the Multi Metal Index $\mathrm{MMI}_{1990-2005}$. The $\mathrm{N}$ concentration reaches from 1.08 to 2,29\% in dry mass showing significant correlations to the agriculture density $(+)$, the height of the surrounding trees $(+)$, the forests density $(-)$, the distance to trees $(-)$, altitude $(-)$ and the precipitation sum for the (Continued on next page)
} 


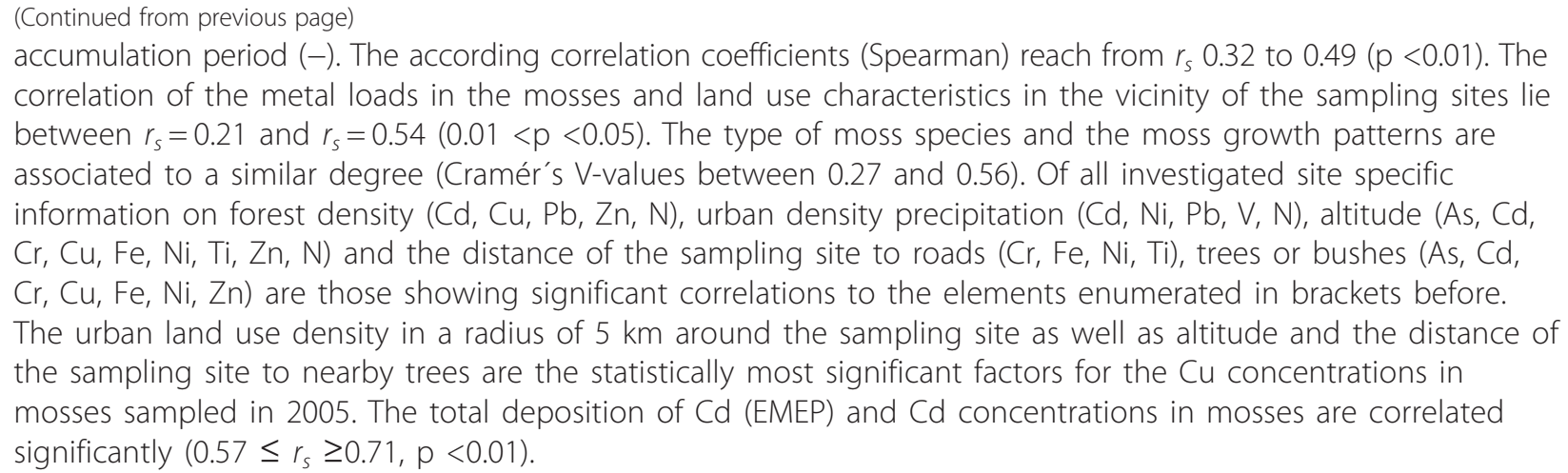

\section{Hintergrund und Ziel}

Umweltmonitoring ist Kernbestandteil der Agenda 21 sowie der Nachhaltigkeitsstrategien des UNESCOProgramms Man and the Biosphere $(\mathrm{MaB})$ und der UNECE (http://www.unece.org/env/welcome.html). Die Langfristperspektive des Nachhaltigkeitsleitbildes bedeutet im Zusammenhang mit der Umweltbeobachtung, dass diese auf langjährigen Messnetzen fußen muss. Das MoosMonitoring ist ein solches Langfristmessprogramm, in dem die Anreicherung von Metallen und Stickstoff in biologischem Material europaweit bestimmt wird. Es wird von der UNECE im International Cooperative Programme on Effects of Air Pollution on Natural Vegetation and Crops (ICP Vegetation) auf Grundlage des Genfer Luftreinhalteabkommens (Convention on Long-Range Transboundary Air Pollution, CLRTAP) im Fünfjahresturnus an mindestens 1.5 Standorten pro $1000 \mathrm{~km}^{2}$ europaweit durchgeführt. Die Ergebnisse werden in Berichten der Working Group on Effects (WGE) der CLRTAP und des Programmzentrums des ICP Vegetation veröffentlicht [1].

Moose werden jedoch nicht nur im kontinentalen Maßstab für das Monitoring der Bioakkumulation atmosphärischer Depositionen eingesetzt, sondern auch lokal - auf einzelne Emittenten bezogen - sowie regional [2-16]. Deutschland beteiligte sich 1990, 1995, 2000 und 2005 an den Heavy Metals in Mosses Surveys, 2010 hingegen nicht. Dabei wirkten Bund und Länder zusammen: Im Auftrag des Umweltbundesamtes (UBA) erfolgte die chemische Analyse der Moose und die Datenauswertung, die Bundesländer übernahmen die Moosprobenentnahme. Der Arbeitskreis Bioindikation / Wirkungsermittlung der Landesämter und -anstalten für Umweltschutz (AKB) und ein Vertreter des UBA begleiteten das MoosMonitoring als wissenschaftlicher Beirat. Die Daten des Moos-Monitorings sollten nicht nur für das gesamte Untersuchungsgebiet, sondern auch als Fallstudien für ausgewählte Bundesländer analysiert werden. Dieser Artikel behandelt eine der Fallstudien, nämlich die Kartierung der Metallanreicherung in Nordrhein-Westfalen seit 1990 und der für die Eutrophierung terrestrischer Ökosysteme wichtigen Stickstoffanreicherung 2005, die Korrelation von Cd-Konzentrationen in den Moosen und in der modellierten Cd-Gesamtdeposition (EMEP) $[17,18]$ sowie die räumliche Varianz der $\mathrm{Cu}$-Bioakkumulation in Abhängigkeit von Eigenschaften der Probeentnahmestellen und ihrer Umgebung. Nordrhein-Westfalen zeichnete sich in allen vier Kampagnen durch überdurchschnittlich hohe $\mathrm{Cd}$ - und $\mathrm{Cu}-$ Konzentrationen in den Moosen aus.

\section{Methoden \\ Datenerhebung}

Das Moos-Monitoring wurde in Deutschland 1990, 1995, 2000 und 2005 nach der europaweit verwendeten Richtlinie [19] durchgeführt. In Nordrhein-Westfalen wurden Moose an 55 (1990), 84 (1995), 88 (2000) bzw. 81 Standorten eingesammelt und die Massenkonzentrationen der Elemente Aluminium ( $\mathrm{Al})$, Barium (Ba), Calcium (Ca), Kupfer (Cu), Eisen (Fe), Kalium (K), Magnesium (Mg), Mangan (Mn), Natrium (Na), Strontium $(\mathrm{Sr})$, Titan (Ti) und Zink (Zn) mit ICP-OES nach DIN EN ISO 11885 (E 22) und VDLUFA Methodenbuch 2.2.2.6 gemessen. Die Massenkonzentrationen der Elemente Arsen (As), Cadmium (Cd), Kobalt (Co), Chrom $(\mathrm{Cr})$, Molybdän (Mo), Nickel (Ni), Blei $(\mathrm{Pb})$, Antimon (Sb) und Vanadium (V) wurden mit ICP-MS nach DIN 38406-29 (E 29) und VDLUFA Methodenbuch 2.2.2.5 bestimmt, die Gehalte von Quecksilber $(\mathrm{Hg})$ in den Moosen mit einem Quecksilberanalysator (Thermostatisierte Zweiwegzelle) nach DIN EN 1483 und VDLUFA Methodenbuch VII. Die Massenkonzentration vom Gesamt-N (Gesamtstickstoff) wurde mit einem C/NAnalyser (Wärmeleitfähigkeitsdetektor) nach VDLUFA Methodenbuch II 3.5.2.7 durch die Verbrennung von 0,2 g Moosprobenmaterial im Sauerstoffstrom ermittelt.

Die Qualität der Messungen wurde zunächst in den einzelnen Teilnehmerstaaten kontrolliert und umfasste die Messung der Referenzmaterialien M2 (hohe Metallkonzentrationen) und M3 (Hintergrundwerte) [20] in 
jeder Messserie sowie den Austausch und die Messung von Moosproben, die auf beiden Seiten der Grenzen Deutschlands zu benachbarten Staaten gesammelt wurden. Anschließend wurden alle qualitätskontrollierten Daten der Teilnehmerstaaten vom Programmzentrum des ICP Vegetation erneut daraufhin überprüft, ob sie den Anforderungen nach [20] entsprechen. In den deutschen Moos-Monitoring-Kampagnen wurden zur Sicherung und Kontrolle der analytischen Qualität alle einschlägigen Maßnahmen [21] ergriffen und ausführlich dargestellt [22]. Dies schließt auch die Analyse von Rückstellproben mit ein, um so die Vergleichbarkeit der Ergebnisse von zwei aufeinander folgenden Kampagnen zu überprüfen. Bevor die qualitätskontrollierten Daten von den Teilnehmerstaaten an das Programmzentrum zur Plausibilitätsprüfung aller europaweit gemessenen Daten geschickt wurden, erfolgte eine Plausibilitätsprüfung in jedem Teilnehmerstaat und ggf. der Ausschluss einzelner Datensätze von der Auswertung. Von der Auswertung der in Nordrhein-Westfalen erhobenen Daten wurden so insgesamt 12 Messwerte ausgeschlossen [23].

\section{Datenauswertung}

Die Kartierung der räumlichen Muster der Bioakkumulation einzelner Metalle oder des Metalle übergreifenden Multi-Metall-Index (MMI, s.u.) erfolgte für den bundesweiten Datensatz geostatistisch sowie perzentilstatistisch [23,24]. In einem ersten Schritt wurde dabei die räumliche Autokorrelation der Messwerte untersucht und modelliert [25-27]. Die darauf basierende Extrapolation der Messwerte erfolgte mit Kriging-Verfahren, die Qualität der dazu berechneten Flächenschätzungen wurde per Kreuzvalidierung bestimmt. Der Multi-Metall-Index (MMI) wurde für die standortspezifischen Messwerte und für die aus ihnen berechneten flächenhaften in einer Auflösung von $3 * 3 \mathrm{~km}^{2}$ vorliegenden Schätzwerte berechnet, indem jeweils pro Element 10 Perzentilklassen gebildet und jedem Perzentil dann ein Indexwert zwischen 1 (niedrige Akkumulation) und 10 (hohe Anreicherung) zugewiesen wurde. Der MMI wurde dabei für das gesamte Bundesgebiet berechnet und nachträglich für Nordrhein-Westfalen im GIS ausgestanzt. Der MMI $1990-2005$ eines jeden Moossammelortes oder einer jeden Rasterzelle entspricht dem Durchschnitt der dort ermittelten elementspezifischen Indexwerte der Konzentrationen von $\mathrm{As}, \mathrm{Cd}, \mathrm{Cr}, \mathrm{Cu}, \mathrm{Fe}$, $\mathrm{Ni}, \mathrm{Pb}, \mathrm{V}$, Ti und Zn, die durchgängig 1990, 1995, 2000 und 2005 in Nordrhein-Westfalen gemessen wurden.

Alle Ergebnisse und weiterführende Informationen der Moos-Monitoring-Kampagnen wurden im WebGIS MossMet dokumentiert und sind Bund, Ländern sowie dem ICP Vegetation zugänglich [28,29]. Die elementspezifischen Messdaten der Monitoring-Kampagnen 1990, 1995, 2000 und 2005 sowie die daraus berechneten MMI konnten daher im Hinblick auf folgende Informationen korrelationsstatistisch untersucht werden: 1. standortspezifische Informationen (s.u.) im Sinne der Verfahrensrichtlinie [19], ökologische und topografische Standorteigenschaften, Ablauf und meteorologische Randbedingungen der Moosprobenentnahme, 2. flächenhafte Informationen über das Klima, die Höhenlage über NN, Flächenanteile forstlicher, agrarischer und urbaner Landnutzung nach CORINE Land Cover [30], Entfernung zu großen Industriebetrieben sowie Depositionen in der Umgebung der Moos-MonitoringStandorte. Hierbei handelt es sich um Daten über atmosphärische Depositionen aus dem EMEP-Luftmessnetz des Umweltbundesamtes (wet only), aus dem ICP ForestMessnetz (Freiland- und Bestandesdeposition) sowie Flächenschätzungen der modellierten Nass-, Trocken- und Gesamtdeposition (EMEP).

Stärke und Richtung der statistischen Zusammenhänge zwischen a) jeweils zwei Metallgehalten in den Moosen, b) zwischen Metallkonzentrationen in Moosen und Depositionen (bulk und wet only Freiland, bulk Kronentraufe) und c) zwischen Metallgehalten und modellierter Nass-, Trocken- und Gesamtdeposition (EMEP) wurden durch Rangkorrelationskoeffizienten nach Spearman $r_{s}$ quantifiziert. Die Korrelation der Daten aus dem EMEP und dem Moosmonitoring erfolgte anhand des Medians der Metallgehalte in Moosen und Depositionen innerhalb der jeweiligen $50 \times 50 \mathrm{~km}^{2}$ großen EMEP-Gridzellen. Zusätzlich wurden multivariat-statistische Korrelationsmuster mit Classification and Regression Trees (CART) [31] berechnet. Dabei bildeten die $\mathrm{Cu}$-Gehalte der Moose 2005 die Zielvariable. Als Prädiktoren dienten die standortbeschreibenden Metainformationen sowie flächenhaft vorliegende Daten zur Umgebungsbeschreibung.

Zur Charakterisierung der Immissions- bzw. Depositionssituation im Umfeld der Probenentnahmestandorte wurde auf ein $100 * 100 \mathrm{~m}^{2}$ Grid der Corine Landcover 2000 sowie auf einen frei verwendbaren Verkehrsnetzlayer der Firma ESRI zurückgegriffen. Der Straßenlayer wurde in einem ersten Schritt um die in der Probenentnahmerichtlinie deklarierten Mindestabstände zu Autobahnen und Landstraßen $(=300 \mathrm{~m})$ gepuffert, das Ergebnis auf $100 * 100 \mathrm{~m}^{2}$ gerastert und anschließend mit dem Corine Landcover Grid verschnitten. Anschließend wurde in $300 \mathrm{~m}, 1 \mathrm{~km}$ und $5 \mathrm{~km}$ Radien um jede Rasterzelle der prozentuale Flächenanteil gewählter Landnutzungsarten berechnet und auf die entsprechende Rasterzelle projiziert. Dies erfolgte sowohl für urbane Flächennutzungen als auch für agrarisch genutzte Flächen. Zusätzlich wurden entsprechende Kennziffern für verkehrsbeeinflusste Flächen für Radien von $1 \mathrm{~km}$ und $5 \mathrm{~km}$ sowie für Waldnutzungen für einen Radius von $5 \mathrm{~km}$ berechnet [23].

Um die Stickstoffgehalte in den Moosen um den Traufeeffekt zu bereinigen, erfolgte eine Umrechnung der 
Stickstoffakkumulationen mit den Schwefelgehalten in den Moosen. Die S-Konzentrationen in Moosen variieren bei gegenwärtig niedrigen $\mathrm{SO}_{2}$-Immissionen großräumig nur gering [8]. Unterschiede der S-Gehalte sind somit in großen Teilen Deutschlands vorrangig auf abweichende Depositionsbedingungen am Probenentnahmeort zurückzuführen. Aufgrund der hohen Korrelation sedimentierender S- und N-Einträge im Niederschlag [32] sowie solcher in den untersuchten Moospflanzen (2005: $\mathrm{r}=0.83 ; \mathrm{p}<0,0001$ ) kann der S-Gehalt als Indikator für den Traufeinfluss der Begleitvegetation (vornehmlich Bäume und Sträucher) und zur Standardisierung der $\mathrm{N}$-Gehalte herangezogen werden.
Die Umrechnung basiert auf der folgenden empirischen Formel: $\mathrm{N}_{\text {korrigiert }}=[1000 / \mathrm{S}-$ Konzentration $] *$

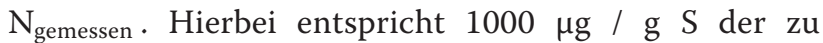
erwartenden S-Bioakkumulation unter Freilandbedingungen außerhalb des Kronentrauf-Bereiches.

\section{Ergebnisse und diskussion}

1990 war Pleurozium schreberi die am häufigsten gesammelte Moosart in Nordrhein-Westfalen, gefolgt von Scleropodium purum und Hypnum cupressiforme (Abbildung 1, Tabelle 1). Seit 1995 ist Scleropodium die Hauptmoosart mit steigenden Anteilen, die Anteile von Pleurozium sanken kontinuierlich.

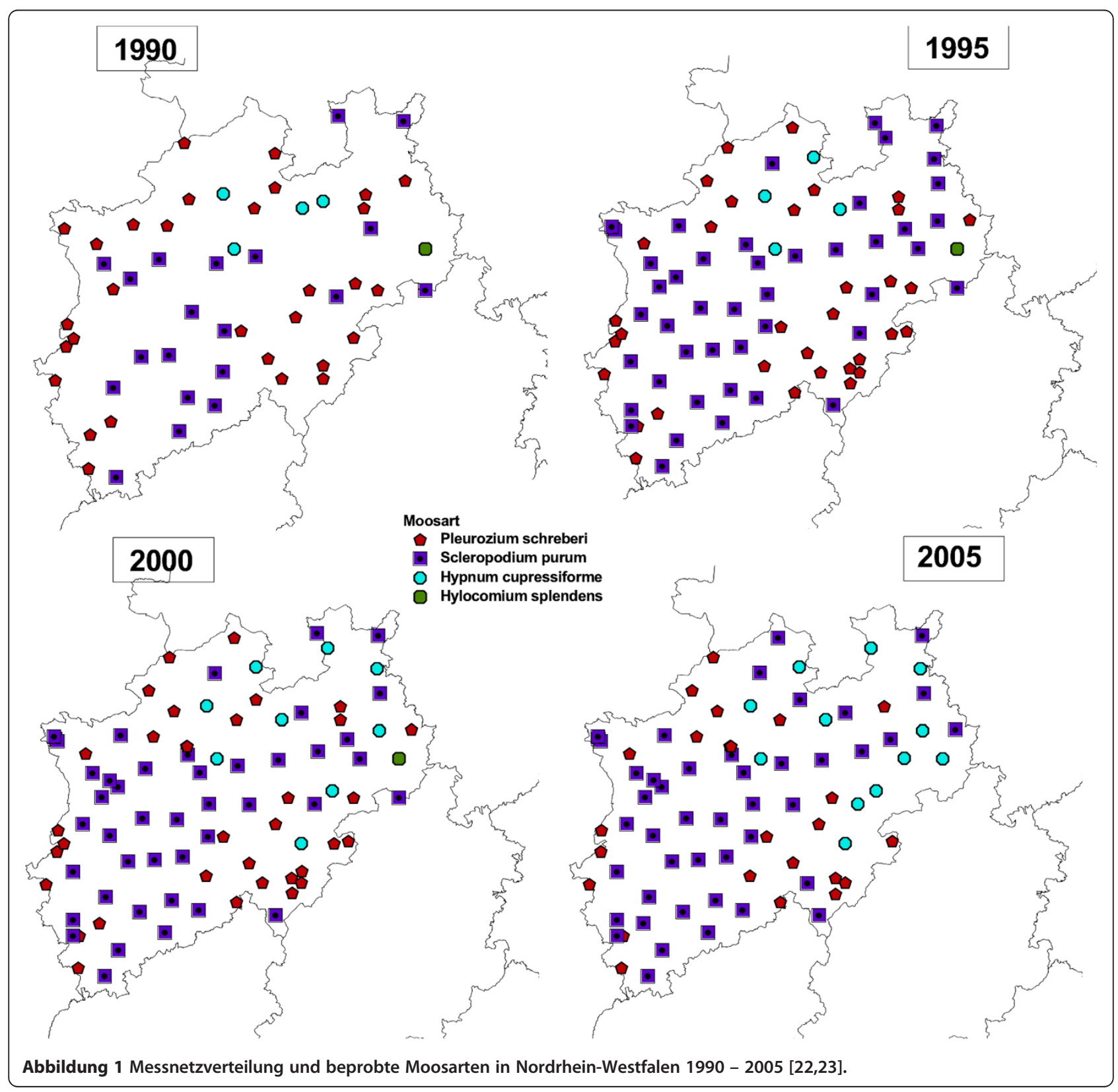


Tabelle 1 Beprobte moosarten in Nordrhein-Westfalen 1990 bis 2005

\begin{tabular}{|c|c|c|c|c|c|c|c|c|}
\hline & \multicolumn{2}{|c|}{ Hypnum cupressiforme } & \multicolumn{2}{|c|}{ Hylocomium splendens } & \multicolumn{2}{|c|}{ Pleurozium schreberi } & \multicolumn{2}{|c|}{ Scleropodium purum } \\
\hline & $n$ & $\%$ & $\mathrm{n}$ & $\%$ & $n$ & $\%$ & $n$ & $\%$ \\
\hline 1990 & 4 & 7.3 & 1 & 1.8 & 30 & 54.5 & 20 & 36.4 \\
\hline 1995 & 4 & 4.8 & 1 & 1.2 & 33 & 39.3 & 46 & 54.8 \\
\hline 2000 & 9 & 10.2 & 1 & 1.1 & 34 & 38.6 & 44 & 50.0 \\
\hline 2005 & 12 & 14.8 & 0 & 0.0 & 24 & 29.6 & 45 & 55.6 \\
\hline
\end{tabular}

Anhand des 20., 50. und 90. Perzentils sowie des MMI lässt sich eine meist signifikante Abnahme der Metallanreicherung in Moosen zwischen 1990 und 2000 nachweisen (Tabelle 2). Auffällig ist, dass die Abnahmen der Mediane bei $\mathrm{Cr}$ und $\mathrm{Zn}$ nicht signifikant sind und sich von 2000 nach 2005 in eine signifikante Zunahme verkehren (Tabelle 3). Einen wenn auch nicht signifikanten - Anstieg weisen As, $\mathrm{Cu}, \mathrm{Ni}, \mathrm{Sb}$ und der MMI auf, während die Gehalte an $\mathrm{Cd}, \mathrm{Fe}, \mathrm{Hg}$, Ti und $\mathrm{V}$ sinken, allerdings nicht signifikant. Eine weiterhin signifikante Reduzierung ist für $\mathrm{Pb}$ gemessen worden.

Die räumliche Differenzierung der in den Tabellen 2 und 3 quantitativ beschriebenen Entwicklung der
Stoffanreicherung wird am Beispiel des $\mathrm{MMI}_{1990-2005}$ in Abbildung 2 kartografisch veranschaulicht. Die Abweichungen zwischen den auf Schätzungen basierenden $\mathrm{MMI}_{1990-2005}$ (8.0, 7.0, 3.2, 4.1) (Abbildung 2) von den MMI, die auf den exakten Messwerten beruhen (7.8, 6.7, 4.0, 4.2) (Tabelle 2), ist auf Abweichungen der interpolierten von den gemessenen Werten zurückzuführen. Beide $\mathrm{MMI}_{1990-2005}$ liegen im Vergleich zum Bundesgebiet in allen vier Kampagnen höher: Aus den rasterbasierten $\mathrm{MMI}_{1990-2005}$ lassen sich bundesweite Mediane von 7.9 (1990), 6.6 (1995), 3.1 (2000) sowie 3.9 (2005) berechnen, aus den messdatenbasierten $\mathrm{MMI}_{1990-2005}$ ergeben sich Mediane von 7.7 (1990), 6.5 (1995), 3.9 (2000) sowie 3.4 (2005) [23]. Das räumliche Muster der

Tabelle 2 Deskriptiv-statistische Maßzahlen der Elementgehalte in nordrhein-westfälischen Moosen 1990 - 2005 (Perzentilangaben für die Elemente in $\mu \mathrm{g} / \mathrm{g}$ )

\begin{tabular}{|c|c|c|c|c|c|c|c|c|c|c|c|c|c|}
\hline & As & Cd & $\mathrm{Cr}$ & $\mathrm{Cu}$ & $\mathrm{Fe}$ & $\mathrm{Hg}$ & $\mathrm{Ni}$ & $\mathrm{Pb}$ & $\mathrm{Sb}$ & $\mathrm{Ti}$ & V & $\mathrm{Zn}$ & MMI \\
\hline \multicolumn{14}{|c|}{1990} \\
\hline Anzahl & 55 & 55 & 55 & 55 & 55 & & 55 & 55 & & 55 & 55 & 55 & 55 \\
\hline 20. Perz & 0.290 & 0.364 & 2.24 & 8.77 & 561 & & 2.27 & 13.67 & & 22.5 & 2.91 & 51.7 & 6.52 \\
\hline 50. Perz & 0.358 & 0.476 & 3.11 & 10.38 & 735 & & 2.87 & 17.40 & & 26.2 & 3.97 & 66.2 & 7.8 \\
\hline 90. Perz & 0.607 & 0.672 & 4.82 & 16.02 & 1144 & & 4.76 & 25.90 & & 41.2 & 5.40 & 105.7 & 9.24 \\
\hline \multicolumn{14}{|c|}{1995} \\
\hline Anzahl & 84 & 84 & 84 & 84 & 84 & 84 & 84 & 84 & 84 & 84 & 84 & 84 & 84 \\
\hline 20. Perz & 0.100 & 0.378 & 1.61 & 10.70 & 351 & 0.048 & 1.50 & 7.37 & 0.183 & 13.8 & 1.50 & 66.0 & 4.7 \\
\hline 50. Perz & 0.165 & 0.501 & 2.51 & 12.98 & 502 & 0.062 & 2.10 & 11.80 & 0.244 & 17.8 & 2.13 & 80.6 & 6.7 \\
\hline 90. Perz & 0.404 & 0.761 & 6.80 & 19.70 & 937 & 0.107 & 3.60 & 21.80 & 0.418 & 30.9 & 3.43 & 125.0 & 9.2 \\
\hline \multicolumn{14}{|c|}{2000} \\
\hline Anzahl & 88 & 88 & 88 & 88 & 88 & 88 & 88 & 88 & 88 & 87 & 87 & 87 & 88 \\
\hline 20. Perz & 0.119 & 0.268 & 1.01 & 7.21 & 306 & 0.039 & 1.16 & 5.24 & 0.160 & 5.3 & 1.01 & 48.4 & 2.5 \\
\hline 50. Perz & 0.160 & 0.353 & 1.32 & 9.20 & 401 & 0.047 & 1.54 & 7.04 & 0.235 & 7.1 & 1.46 & 62.5 & 4 \\
\hline 90. Perz & 0.250 & 0.491 & 1.91 & 13.21 & 575 & 0.074 & 2.53 & 11.08 & 0.391 & 10.8 & 2.05 & 96.8 & 5.95 \\
\hline \multicolumn{14}{|c|}{2005} \\
\hline Anzahl & 80 & 79 & 80 & 80 & 81 & 81 & 81 & 80 & 80 & 81 & 81 & 79 & 81 \\
\hline 20. Perz & 0.120 & 0.260 & 1.51 & 7.51 & 274 & 0.035 & 1.11 & 4.23 & 0.162 & 4.1 & 0.98 & 51.9 & 3 \\
\hline 50. Perz & 0.180 & 0.315 & 2.44 & 9.65 & 379 & 0.043 & 1.72 & 6.16 & 0.243 & 6.5 & 1.45 & 73.3 & 4.2 \\
\hline 90. Perz & 0.338 & 0.585 & 5.36 & 16.21 & 725 & 0.058 & 2.68 & 11.39 & 0.399 & 12.2 & 2.26 & 123.0 & 7.18 \\
\hline
\end{tabular}


Tabelle 3 Inferenz-statistische analyse der Entwicklung der Stoffakkumulationen in nordrhein-westfälischen Moosen 1990 - 2005 (bezogen auf die mediandifferenzen, verwendet wurde der signifikanztest nach Mann und Whitney ungepaarte Stichproben)

\begin{tabular}{cccccccccccccc}
\hline & As & Cd & Cr & Cu & Fe & Hg & Ni & Pb & Sb & Ti & V & Zn & MMI \\
\hline $1990-1995$ & A & Z & A & Z & A & & A & A & & A & A & Z & A \\
$1990-2000$ & A & A & A & A & A & & A & A & & A & A & A & A \\
$1990-2005$ & A & A & A & A & A & & A & A & & A & A & Z & A \\
$1995-2000$ & A & A & A & A & A & A & A & A & A & A & A & A & A \\
$1995-2005$ & Z & A & A & A & A & A & A & A & A & A & A & A & A \\
$2000-2005$ & Z & A & Z & Z & A & A & Z & A & Z & A & A & Z & Z
\end{tabular}

Fett: $\quad$ Signifikante Änderung $(A=A b n a h m e ; Z=$ Zunahme; $p<0.01)$

FettKursiv: $\quad$ Signifikante Änderung $(A=A b n a h m e ; Z=$ Zunahme; $0,01<p>0,05)$

Normal: $\quad$ Keine signifikante Zu- oder Abnahme

Stickstoffanreicherung wird anhand der OriginalMessdaten sowie den mit der Schwefelformel (Abschnitt 2.2) umgerechneten Werten dargestellt (Abbildung 3). Nordrhein-Westfalen zeigt den höchsten Median (1.8\%) aller Bundesländer [23].
In Ergänzung zu den bei der Moosproben-Entnahme erhobenen Informationen über Eigenschaften der Sammelorte wurden auch flächenhafte Daten berücksichtigt, welche die Landnutzung und insofern potenzielle Flächenquellen für Metalle und Stickstoff in ihrer

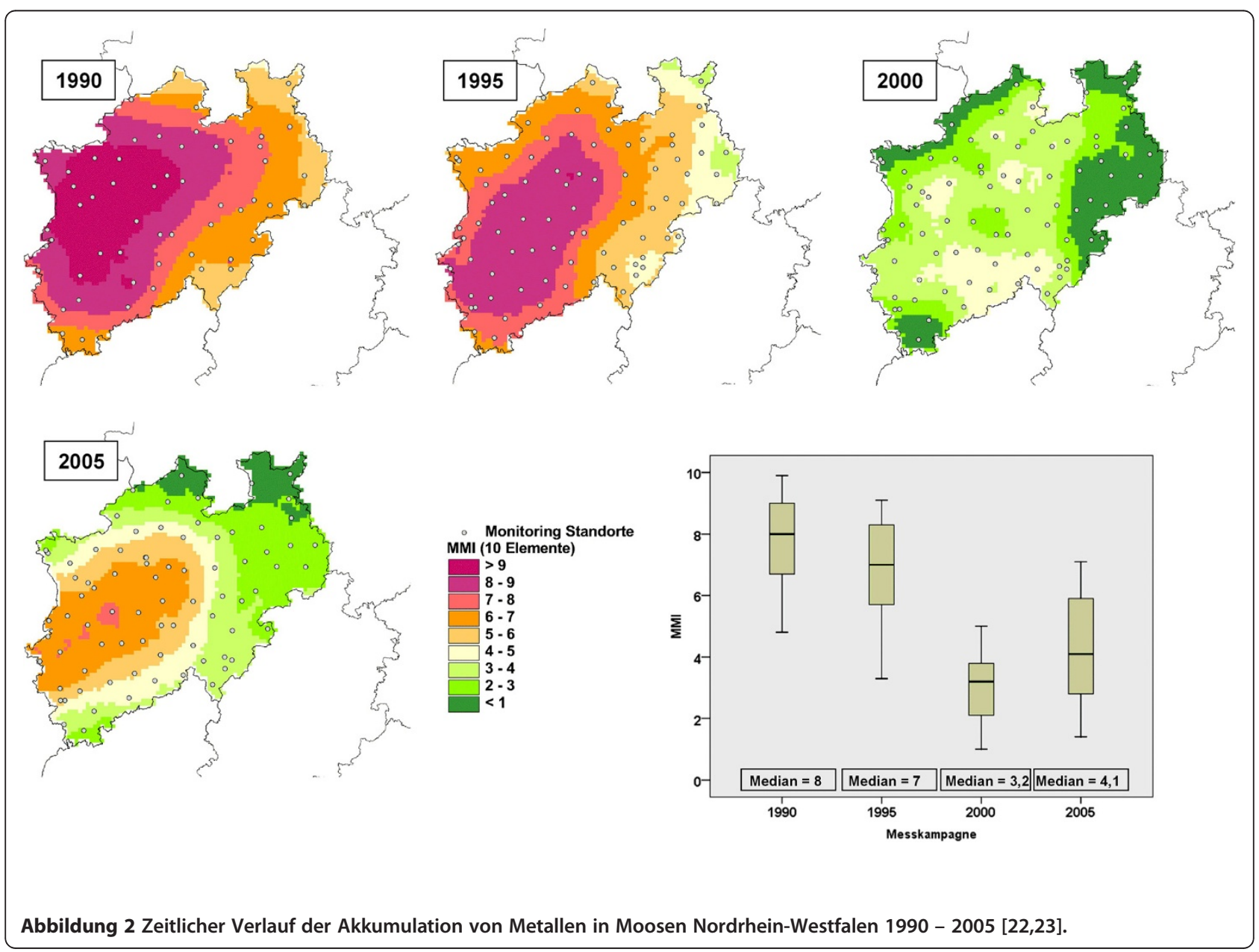




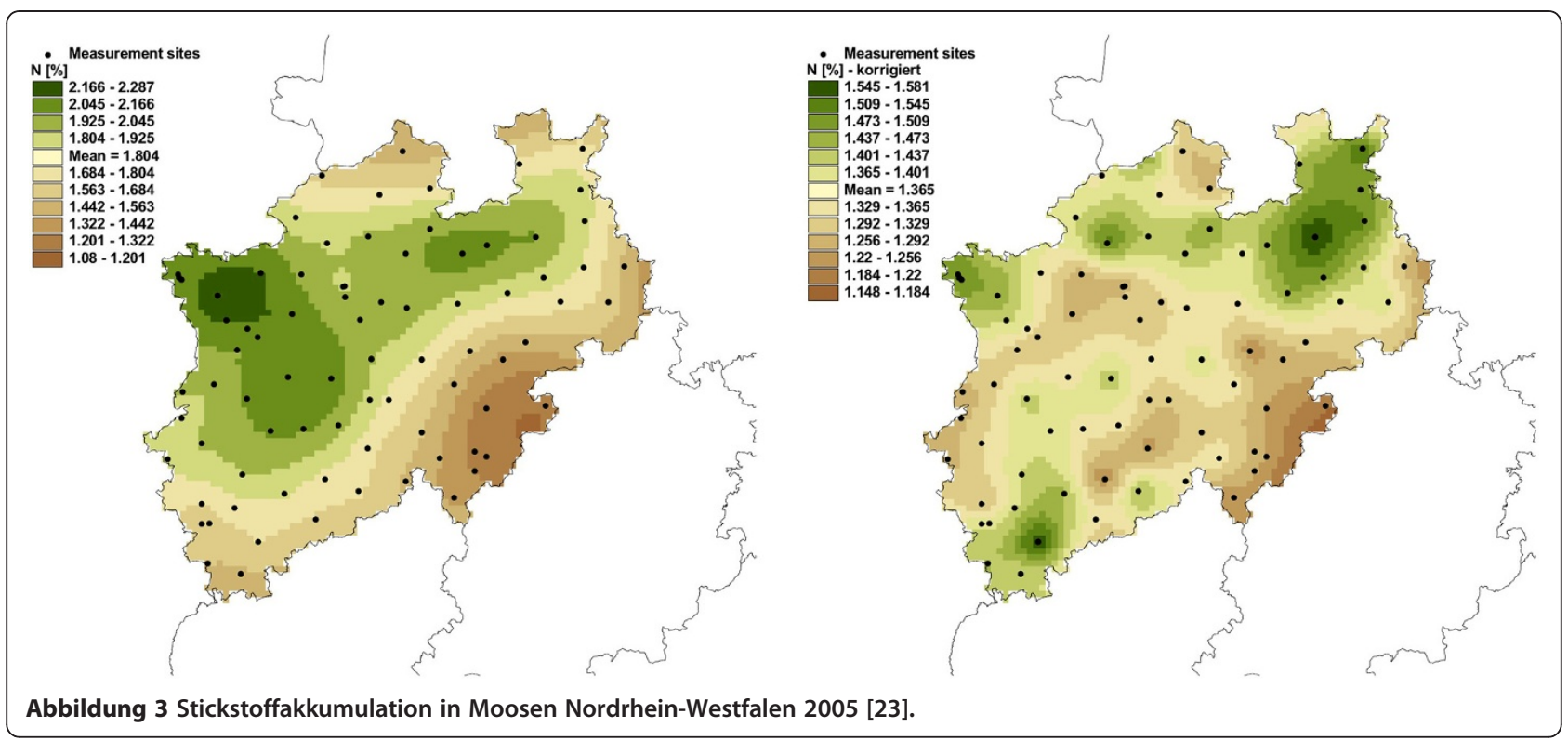

Umgebung kennzeichnen [23]. Die Ergebnisse der bivariaten Zusammenhangsanalysen werden für metrische Daten in den Tabellen 4 und 5 in Form des Rangkorrelationskoeffizienten nach Spearman $\left(r_{s}\right)$ präsentiert. Die Stickstoffgehalte sind positiv mit dem Agrarflächenanteil im Umkreis der Beprobungsstellen und der Bestandeshöhe sowie negativ mit der Entfernung zu Bäumen und zur Höhe über NN korreliert $\left(0.32 \leq \mathrm{r}_{s} \geq 0.49, \mathrm{p}<0.01\right)$.

Da besonders die Agrarproduktion $\mathrm{zu}$ erhöhten $\mathrm{N}$ Konzentrationen in der Atmosphäre beiträgt und ferner die Bestandeshöhe und die Entfernung zu Bäumen den Filtereffekt der umgebenden Vegetation widerspiegeln, sind diese Ergebnisse plausibel. Gleiches gilt für die orografische Höhe, die positiv korreliert mit dem Niederschlag, was zu verstärktem Wachstum der Moose und somit zu Verdünnungseffekten führen kann. Die Zusammenhänge zwischen Metallgehalten in den Moosen und der Landnutzung im Umkreis der Beprobungsorte werden durch Werte zwischen $\mathrm{r}_{s}=0.21$ und $\mathrm{r}_{s}=0.54(0.01$ $<\mathrm{p}<0.05)$ charakterisiert. Moosart und -bewuchs sind mit den Stoffkonzentrationen ähnlich stark assoziiert (Cramér's V-Werte zwischen 0.27 und 0.56; Tabelle 6).

Von den die Moossammelstellen charakterisierenden Merkmalen weisen vor allem der Flächenanteil urbaner Landnutzung (bei As, $\mathrm{Cd}, \mathrm{Cr}, \mathrm{Cu}, \mathrm{Fe}, \mathrm{Ni}, \mathrm{Ti}, \mathrm{Zn}$ ), die Niederschlagssumme (bei $\mathrm{Cd}, \mathrm{Ni}, \mathrm{Pb}, \mathrm{V}, \mathrm{N}$ ), die orografische Höhe (alle außer bei $\mathrm{Hg}, \mathrm{Pb}, \mathrm{Sb}, \mathrm{V}$ ) sowie die Entfernung der Moos-Entnahmestelle von Straßen (bei $\mathrm{Cr}, \mathrm{Fe}, \mathrm{Ni}$, $\mathrm{Ti}$ ), Baumkronen oder Sträuchern (bei As, Cd, Cr, Cu, $\mathrm{Fe}, \mathrm{Ni}, \mathrm{Zn}$ ) für die meisten Elemente signifikante Korrelationen zur Metallanreicherung in den Moosen auf. Entsprechend den N-Konzentrationen in den Moosen spiegeln sich hier somit v.a. die umgebende Landnutzung wie auch der Filtereffekt der Bäume in den Metallkonzentrationen wider. Bis auf $\mathrm{Ni}, \mathrm{Pb}$ und $\mathrm{V}$ weisen die Korrelationskoeffizienten der Metallelemente mit den Niederschlagssummen negative Werte auf, was auf die tendenzielle Auswaschung dieser Elemente aus den Moosen hindeutet.

Multivariat-statistische Zusammenhänge zwischen der Cu-Bioakkumulation im Jahr 2005 mit lokalen und regionalen Raummerkmalen werden in Abbildung 5 gezeigt. Knoten 0 beschreibt die Verteilung der $\mathrm{Cu}$ Messwerte an 80 Standorten, die im Jahr 2005 in Nordrhein-Westfalen beprobt wurden. Der Mittelwert der Untersuchungsstichprobe beträgt $9.85 \mu \mathrm{g} \mathrm{Cu} \mathrm{/} \mathrm{g}$ Trockensubstanz Moos. Der urbane Flächenanteil im Umkreis von $10 \mathrm{~km}$ um die Moossammelorte ist von den in Tabelle 4 gelisteten Faktoren derjenige, der mit den $\mathrm{Cu}$-Konzentrationen in den Moosen am engsten verknüpft ist: Beträgt der Anteil urbaner Flächennutzung in der Umgebung der Moossammelstellen mehr als $33.6 \%$, beträgt die $\mathrm{Cu}$-Konzentration in den gesammelten Moosproben im Mittel $13.60 \mu \mathrm{g} / \mathrm{g}$. Unterhalb von 33.6\% urbaner Flächennutzung beträgt die mittlere $\mathrm{Cu}$ Konzentration $9.08 \mu \mathrm{g} / \mathrm{g}$. Diese Aufteilung des Gesamtdatensatzes in zwei Untergruppen zeigt sich durchaus plausibel, da höhere $\mathrm{Cu}$-Konzentrationen in den Moosen tendenziell dort zu finden sind, wo der urbane Flächenanteil im Umfeld der Mosssammelstellen hoch ist. Eine Differenzierung dieses Wertes ergibt sich unter dem Einfluss der Höhenlage über NN: Die Cu-Gehalte belaufen sich auf $9.81 \mu \mathrm{g} / \mathrm{g}$ in Höhenlagen unterhalb $333.5 \mathrm{~m}$, hingegen 7.21 $\mu \mathrm{g} / \mathrm{g}$ an Standorten oberhalb 333,5 m. 
Tabelle 4 Korrelationsanalytische Untersuchung von Einflussfaktoren auf die As-, Cd-, Cr-, Cu-, Fe- und Hg-Konzentration in nordrhein-westfälischen Moosen 1990 - 2005. Gezeigt sind signifikante Korrelationskoeffizienten nach Spearman

\begin{tabular}{|c|c|c|c|c|c|c|c|c|c|c|c|c|c|c|c|c|c|c|c|c|c|c|c|c|}
\hline & \multicolumn{4}{|c|}{ As } & \multicolumn{4}{|c|}{$\mathrm{Cd}$} & \multicolumn{4}{|c|}{$\mathrm{Cr}$} & \multicolumn{4}{|c|}{$\mathrm{Cu}$} & \multicolumn{4}{|c|}{$\mathrm{Fe}$} & \multicolumn{4}{|c|}{$\mathrm{Hg}$} \\
\hline & 90 & 95 & 00 & 05 & 90 & 95 & 00 & 05 & 90 & 95 & 00 & 05 & 90 & 95 & 00 & 05 & 90 & 95 & 00 & 05 & 90 & 95 & 00 & 05 \\
\hline $\begin{array}{l}\text { Waldflächenanteilim } \\
5 \text { km Radius [\%] }\end{array}$ & $x$ & -0.27 & & & $x$ & & -0.29 & & $x$ & & & & $x$ & & -0.31 & -0.32 & $x$ & -0.23 & & & $x$ & & & \\
\hline $\begin{array}{l}\text { Verkehrseinflussim } \\
300 \text { m Radius [\%] }\end{array}$ & $x$ & & -0.24 & & $x$ & & & & $x$ & & & & $x$ & & & & $x$ & & & & $x$ & & & \\
\hline $\begin{array}{l}\text { Verkehrseinflussim } 1 \\
\text { km Radius [\%] }\end{array}$ & $X$ & & -0.25 & & $X$ & 0.22 & & & $x$ & & & & $x$ & & & 0.25 & $x$ & & & & $X$ & & & \\
\hline $\begin{array}{l}\text { Verkehrseinflussim } 5 \\
\text { km Radius [\%] }\end{array}$ & $x$ & & & & $x$ & 0.28 & & 0.24 & $x$ & & & & $x$ & & & 0.35 & $x$ & & & & $x$ & & & \\
\hline $\begin{array}{l}\text { Urbaner } \\
\text { Flächenanteil im } 300 \\
\text { m Radius [\%] }\end{array}$ & $x$ & & & & $x$ & 0.37 & & & $x$ & 0.25 & & 0.29 & $x$ & 0.29 & & & $x$ & 0.32 & & & $x$ & 0.36 & & \\
\hline $\begin{array}{l}\text { Urbaner } \\
\text { Flächenanteil im } 1 \\
\text { km Radius [\%] }\end{array}$ & $x$ & 0.24 & & & $x$ & 0.33 & & & $x$ & & & 0.34 & $x$ & & 0.23 & & $x$ & 0.26 & & & $x$ & & & \\
\hline $\begin{array}{l}\text { Urbaner } \\
\text { Flächenanteil im } 5 \\
\text { km Radius [\%] }\end{array}$ & $x$ & 0.32 & & & $x$ & 0.47 & 0.28 & 0.32 & $x$ & 0.37 & & 0.33 & $x$ & 0.29 & 0.33 & 0.44 & $x$ & 0.39 & & & $x$ & 0.26 & & \\
\hline $\begin{array}{l}\text { Agrar Flächenanteil } \\
\text { im } 300 \text { m Radius [\%] }\end{array}$ & $X$ & & & & $x$ & & & & $x$ & & & & $x$ & & & & $x$ & & & & $X$ & & & \\
\hline $\begin{array}{l}\text { Agrar Flächenanteil } \\
\text { im } 1 \text { km Radius [\%] }\end{array}$ & $x$ & & & & $x$ & & & & $x$ & & & & $x$ & & & & $x$ & & 0.22 & & $x$ & & & \\
\hline $\begin{array}{l}\text { Agrar Flächenanteil } \\
\text { im } 5 \text { km Radius [\%] }\end{array}$ & $x$ & & & & $x$ & & & & $x$ & & & & $x$ & & & & $x$ & & & & $x$ & & & \\
\hline $\begin{array}{l}\text { Niederschlagssumme } \\
2004-2006[\mathrm{~mm}]\end{array}$ & $x$ & $x$ & $x$ & & $x$ & $x$ & $x$ & & $x$ & $x$ & $x$ & & $x$ & $x$ & $x$ & & $x$ & $x$ & $x$ & & $x$ & $x$ & $x$ & \\
\hline $\begin{array}{l}\text { Niederschlagssumme } \\
2003-2006[\mathrm{~mm}]\end{array}$ & $x$ & $x$ & $x$ & & $x$ & $x$ & $x$ & & $x$ & $x$ & $x$ & & $x$ & $x$ & $x$ & & $x$ & $x$ & $x$ & & $x$ & $x$ & $x$ & \\
\hline $\begin{array}{l}\text { Distanzzur Nord- / } \\
\text { Ostsee }\end{array}$ & $X$ & $X$ & $X$ & $x$ & $x$ & $x$ & $X$ & $x$ & $x$ & $x$ & $X$ & $X$ & $X$ & $x$ & $X$ & $X$ & $x$ & $x$ & $x$ & $x$ & $x$ & $X$ & $X$ & $x$ \\
\hline Höheüber NN [m] & -0.34 & -0.25 & & & -0.42 & -0.22 & -0.30 & & -0.44 & -0.23 & & -0.23 & -0.41 & & -0.32 & -0.31 & -0.42 & -0.28 & & & & & & \\
\hline Neigung & -0.40 & & & & -0.44 & & -0.25 & -0.37 & -0.47 & & & & -0.41 & & -0.26 & & -0.54 & & & & & & & \\
\hline Bestandeshöhe & & & & & & & & & & & & & & & 0.22 & & & & & & & & & \\
\hline $\begin{array}{l}\text { Entfernung z. } \\
\text { Baumkrone [m] }\end{array}$ & $x$ & & & & $x$ & & & & $x$ & & & & $x$ & & -0.25 & -0.42 & $x$ & & & & $x$ & & & -0.35 \\
\hline $\begin{array}{l}\text { Entfernung z. Strauch } \\
\text { [m] }\end{array}$ & $x$ & & -0.26 & & $x$ & & & -0.23 & $x$ & 0.30 & & -0.39 & $\mathrm{x}$ & & & -0.31 & $x$ & 0.29 & & & $x$ & & & \\
\hline
\end{tabular}


Tabelle 4 Korrelationsanalytische Untersuchung von Einflussfaktoren auf die As-, Cd-, Cr-, Cu-, Fe- und Hg-Konzentration in nordrhein-westfälischen Moosen 1990 - 2005. Gezeigt sind signifikante Korrelationskoeffizienten nach Spearman (Continued)

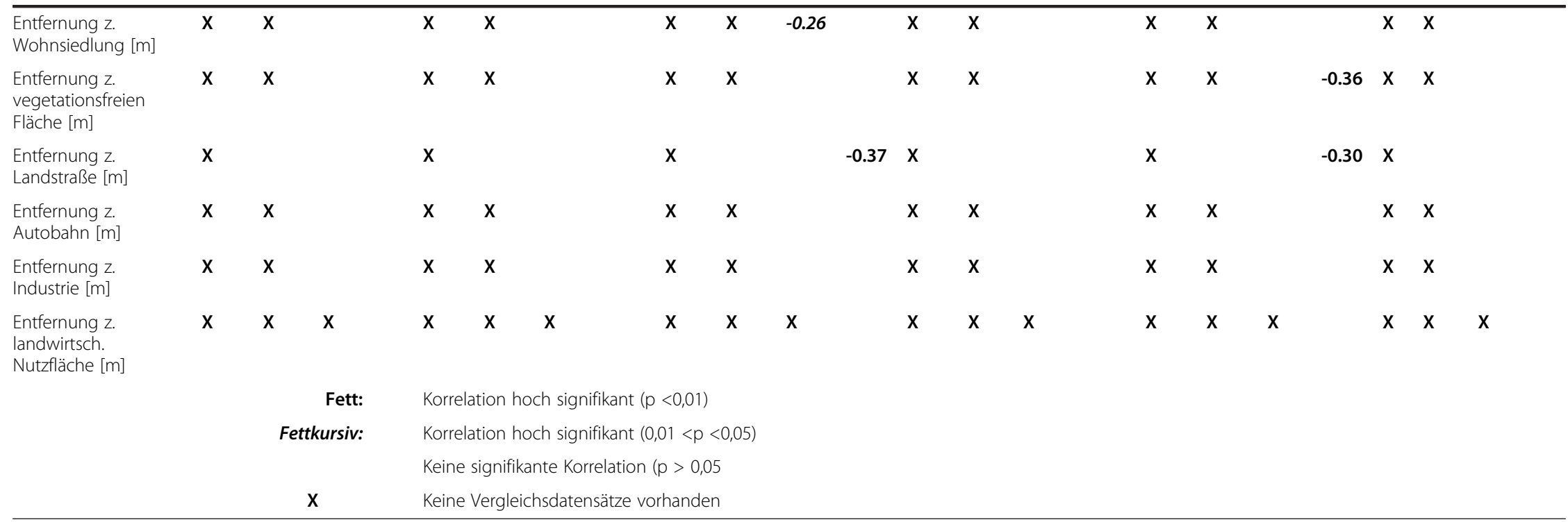


Tabelle 5 Korrelationsanalytische Untersuchung von Einflussfaktoren auf die Ni-, Pb-, Sb-, Ti-, V- und Zn- und N-Konzentration in nordrhein-westfälischen Moosen 1990 - 2005

\begin{tabular}{|c|c|c|c|c|c|c|c|c|c|c|c|c|c|c|c|c|c|c|c|c|c|c|c|c|c|}
\hline & \multicolumn{4}{|c|}{$\mathrm{Ni}$} & \multicolumn{4}{|c|}{$\mathrm{Pb}$} & \multicolumn{4}{|c|}{$\mathrm{Sb}$} & \multicolumn{4}{|c|}{$\mathrm{Ti}$} & \multicolumn{4}{|c|}{ v } & \multicolumn{4}{|c|}{$\mathrm{Zn}$} & \multirow{2}{*}{$\frac{\mathrm{N}}{05}$} \\
\hline & 90 & 95 & 00 & 05 & 90 & 95 & 00 & 05 & 90 & 95 & 00 & 05 & 90 & 95 & 00 & 05 & 90 & 95 & 00 & 05 & 90 & 95 & 00 & 05 & \\
\hline $\begin{array}{l}\text { Waldflächenanteilim } \\
5 \mathrm{~km} \text { Radius [\%] }\end{array}$ & $x$ & & & & $x$ & & & & $x$ & & & & $x$ & & & & $x$ & & & & $x$ & -0.24 & -0.37 & -0.33 & -0.47 \\
\hline $\begin{array}{l}\text { Verkehrseinflussim } \\
300 \text { m Radius [\%] }\end{array}$ & $x$ & & & & $x$ & & & & $x$ & & -0.22 & & $x$ & & & & $x$ & & -0.24 & & $x$ & & & 0.24 & \\
\hline $\begin{array}{l}\text { Verkehrseinflussim } \\
1 \text { km Radius [\%] }\end{array}$ & $x$ & & & & $x$ & & & & $x$ & & & & $x$ & & & & $x$ & & -0.25 & & $x$ & & & 0.30 & \\
\hline $\begin{array}{l}\text { Verkehrseinflussim } \\
5 \text { km Radius [\%] }\end{array}$ & $x$ & & & & $x$ & & & & $x$ & & & & $x$ & 0.24 & & & $x$ & & & & $x$ & & & & \\
\hline $\begin{array}{l}\text { Urbaner } \\
\text { Flächenanteil im } \\
300 \text { m Radius [\%] }\end{array}$ & $x$ & 0.29 & & & $X$ & 0.28 & & & $x$ & 0.29 & & & $x$ & 0.27 & & & $X$ & & & & $x$ & 0.32 & & 0.25 & \\
\hline $\begin{array}{l}\text { Urbaner } \\
\text { Flächenanteil im } \\
1 \text { km Radius [\%] }\end{array}$ & $x$ & & & & $x$ & & & & $x$ & & & & $x$ & 0.25 & & & $x$ & & & & $x$ & 0.25 & 0.22 & 0.25 & \\
\hline $\begin{array}{l}\text { Urbaner } \\
\text { Flächenanteil im } \\
5 \text { km Radius [\%] }\end{array}$ & $X$ & 0.30 & & & $X$ & & & & $x$ & 0.27 & & & $x$ & 0.37 & & 0.26 & $X$ & & & & $x$ & 0.36 & 0.26 & 0.37 & \\
\hline $\begin{array}{l}\text { Agrar Flächenanteil } \\
\text { im } 300 \text { m Radius [\%] }\end{array}$ & $x$ & & & & $x$ & & & & $x$ & & & & $x$ & & & & $x$ & & & & $x$ & & & & \\
\hline $\begin{array}{l}\text { Agrar Flächenanteil } \\
\text { im } 1 \text { km Radius [\%] }\end{array}$ & $X$ & & & & $X$ & & & & $x$ & & & & $x$ & & & & $X$ & & & & $x$ & & & & \\
\hline $\begin{array}{l}\text { Agrar Flächenanteil } \\
\text { im } 5 \text { km Radius [\%] }\end{array}$ & $x$ & -0.31 & -0.34 & -0.24 & $x$ & & & & $x$ & & & & $x$ & & & & $X$ & & & & $x$ & & & & 0.39 \\
\hline $\begin{array}{l}\text { Niederschlagssumme } \\
2004 \text { - } 2006[\mathrm{~mm}]\end{array}$ & $x$ & $x$ & $x$ & 0.28 & $x$ & $x$ & $x$ & 0.23 & $x$ & $x$ & $x$ & & $x$ & $x$ & $x$ & & $x$ & $x$ & $x$ & & $x$ & $x$ & $x$ & & -0.32 \\
\hline $\begin{array}{l}\text { Niederschlagssumme } \\
2003-2006[\mathrm{~mm}]\end{array}$ & $x$ & $x$ & $x$ & 0.27 & $x$ & $x$ & $x$ & & $x$ & $x$ & $x$ & & $x$ & $x$ & $x$ & & $x$ & $x$ & $x$ & 0.22 & $x$ & $x$ & $X$ & & -0.32 \\
\hline $\begin{array}{l}\text { Distanzzur Nord- / } \\
\text { Ostsee }\end{array}$ & $x$ & $x$ & $x$ & $x$ & $x$ & $x$ & $x$ & $x$ & $x$ & $x$ & $x$ & $X$ & $x$ & $x$ & $X$ & $x$ & $X$ & $x$ & $x$ & $X$ & $x$ & $x$ & $X$ & $x$ & $x$ \\
\hline Höheüber NN [m] & & & 0.24 & 0.23 & & & & & $x$ & & & & -0.30 & -0.25 & & & & & & & -0.40 & & -0.27 & -0.27 & -0.39 \\
\hline Neigung & -0.33 & & 0.25 & & -0.31 & & & & $x$ & & & & -0.38 & & & & & & & & -0.51 & & -0.33 & -0.38 & \\
\hline Bestandeshöhe & & & & & & & & & $\mathrm{x}$ & & & & & & & & & & & & & & & & 0.36 \\
\hline $\begin{array}{l}\text { Entfernung z. } \\
\text { Baumkrone [m] }\end{array}$ & $x$ & & & & $x$ & & & & $x$ & & & & $x$ & & & & $x$ & & & & $\mathrm{X}$ & & & & -0.49 \\
\hline $\begin{array}{l}\text { Entfernung z. } \\
\text { Strauch }[\mathrm{m}]\end{array}$ & $x$ & 0.29 & & & $x$ & & & & $\mathrm{x}$ & & & & $x$ & & & & $x$ & & & & $x$ & & & -0.39 & \\
\hline
\end{tabular}


Tabelle 5 Korrelationsanalytische Untersuchung von Einflussfaktoren auf die Ni-, Pb-, Sb-, Ti-, V- und Zn- und N-Konzentration in nordrhein-westfälischen Moosen 1990 - 2005 (Continued)

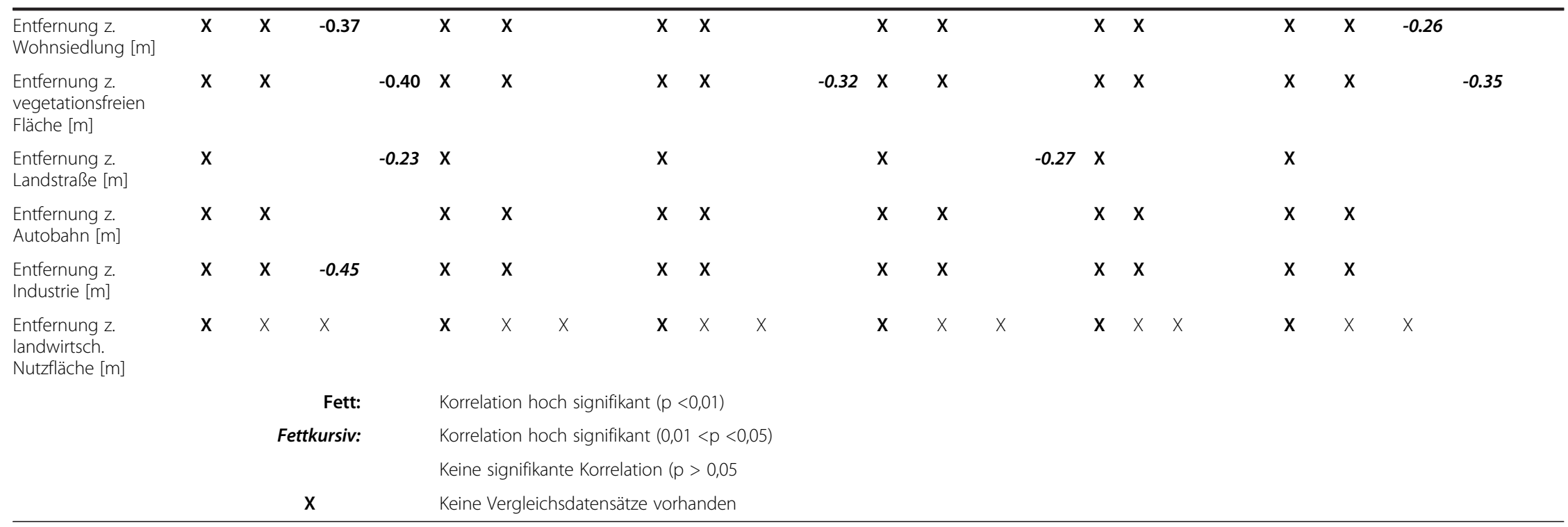


Tabelle 6 Kontingenzanalytische Untersuchung von Einflussfaktoren auf die Stoffakkumulation in nordrheinwestfälischen Moosen 1990 - 2005. Gezeigt sind die signifikanten Cramérs-V Koeffizienten

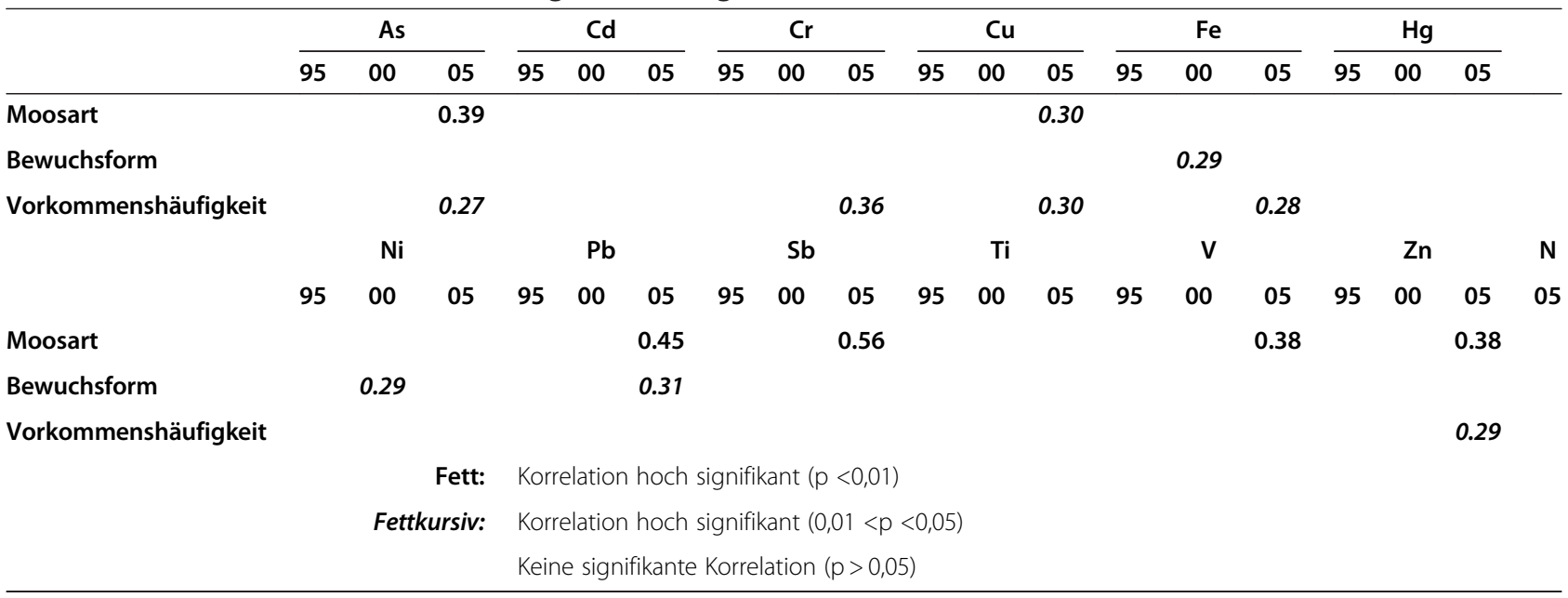

Eine Erklärung hierfür lässt sich aus den Daten nicht ableiten. $\mathrm{Zu}$ denken ist an die Häufigkeit von Nebelereignissen unterhalb und oberhalb dieser Höhengrenze. Nebel hat eine hohe spezifische Oberfläche mit potenziell hohen Stoffgehalten und niedrigen ph-Werten. An Moosbeprobungsorten, in deren Umgebung der Anteil urbaner Flächennutzungen 33.6\% überschreitet, steigen die $\mathrm{Cu}-G e h a l t e$ in Moosen auf $16.2 \mu \mathrm{g} / \mathrm{g}$, wenn die Probe in weniger als $1.5 \mathrm{~m}$ Entfernung zum nächsten Baum genommen wurde. Moose, die in der unmittelbaren Nähe von Bäumen genommen werden, zeigen somit höhere $\mathrm{Cu}$-Werte, was mit dem Filtereffekt der Bäume erklärt werden kann.

In die bivariate Analyse statistischer Zusammenhänge zwischen der Metallanreicherung in Moosen und Eigenschaften der Moossammelorte und ihrer Umgebung wurden EMEP-Daten der Cd-Gesamtdeposition mit einer räumlichen Auflösung von $50 \mathrm{~km}$ mal $50 \mathrm{~km}$ einbezogen (Abbildung 4). Abbildung 5 zeigt im linken Teil die räumliche Differenzierung der $\mathrm{Cd}$-Anreicherungen in den Moosen, wobei die Mediane der Cd-Werte für jedes EMEP-Raster anhand der Einzelmesswerte der in ihm gelegenen Beprobungspunkte berechnet wurden. Im rechten Abbildungsteil ist in derselben räumlichen Auflösung die Cd-Gesamtdeposition dargestellt. Beiden räumlichen Verteilungen sind positive korreliert mit $0.57 \leq r$ $\geq 0.71$ bei $p<0.01$.

Abweichend von den Verhältnissen im Bundesgebiet wurde in Nordrhein-Westfalen die Bioakkumulation von Metallen und Stickstoff am häufigsten in Scleropodium purum bestimmt, gefolgt von Pleurozium schreberi und Hypnum cupressiforme. Eine Umrechnung der Metallkonzentrationen auf jeweils eine Moosart würde nach [33] und [1] Unschärfen erzeugen. Das statistische
Design des Moos-Monitorings ermöglicht es nicht, die mit den verschiedenen Moosarten statistisch assoziierten Metallgehalte so zu interpretieren, dass die Unterschiede alleine durch eine unterschiedliche Sammeleffizienz der Moose bedingt sind. Eine solche Interpretation wäre nur dann zulässig, wenn die miteinander verglichenen Metallgehalte von Moosarten stammen, die an jeweils einem Standort gesammelt wurden. Dies ist jedoch nicht der Fall. Für überregionale Vergleiche in Deutschland und Europa erscheint eine Umrechnung auch deshalb nicht sinnvoll, weil dann eine Nivellierung räumlich differenzierter Hintergrundwerte erfolgen würde. Die Randbedingungen dieser räumlichen Differenzierung zu identifizieren, sollte vielmehr das Ziel des Umweltmonitorings sein. Im Moos-Monitoring gelingt dies flächendeckend, weil in Deutschland und zukünftig auch in anderen europäischen Staaten neben den Messdaten auch wichtige Charakteristika der Moosbeprobungsorte und ihrer Umgebung aufgenommen und in die multivariatstatistischen Auswertungen einbezogen werden.

Die Ergebnisse der multivariat-statistischen Analyse mit CART bestätigen die Ergebnisse der bivariatstatistischen Berechnungen und ergänzen diese um die Identifikation von Kombinationen zwischen Standortsund Umgebungsfaktoren. Die bivariat-statistischen Korrelationsanalysen zeigen am Beispiel $\mathrm{Cd}$, dass sich mit dem Moos-Monitoring flächendeckend und räumlich differenziert für Nordrhein-Westfalen der Nachweis führen lässt, dass abnehmende Cd-Depositionen mit sinkenden Cd-Konzentrationen in Moosen verknüpft sind. Diese auf modellierten Depositionsdaten (EMEP) beruhende Aussage kann bezogen auf das gesamte Bundesgebiet mit Daten aus deutschen Depositionsmessnetzen sowie deutschlandweiten Modellierungen von [34] 

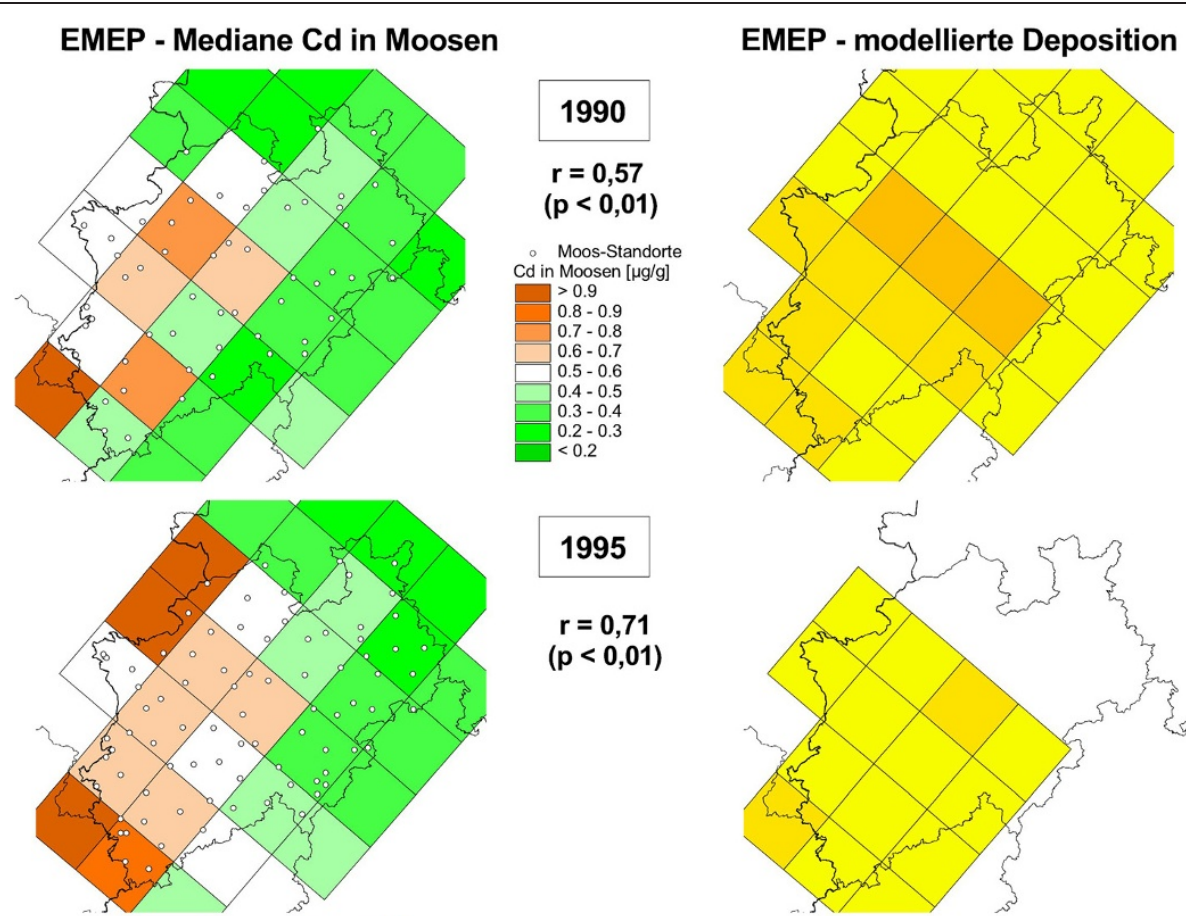

1995

$r=0,71$

$(p<0,01)$

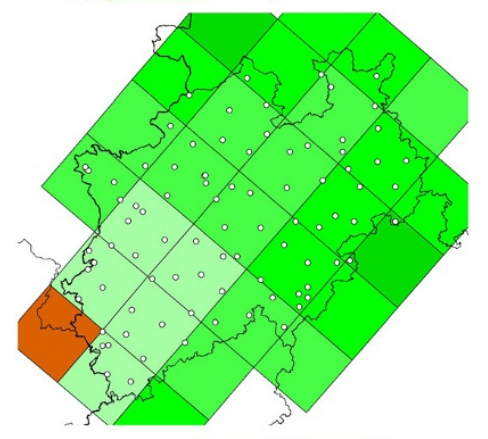

2000

$r=0,66$

$(p<0,01)$
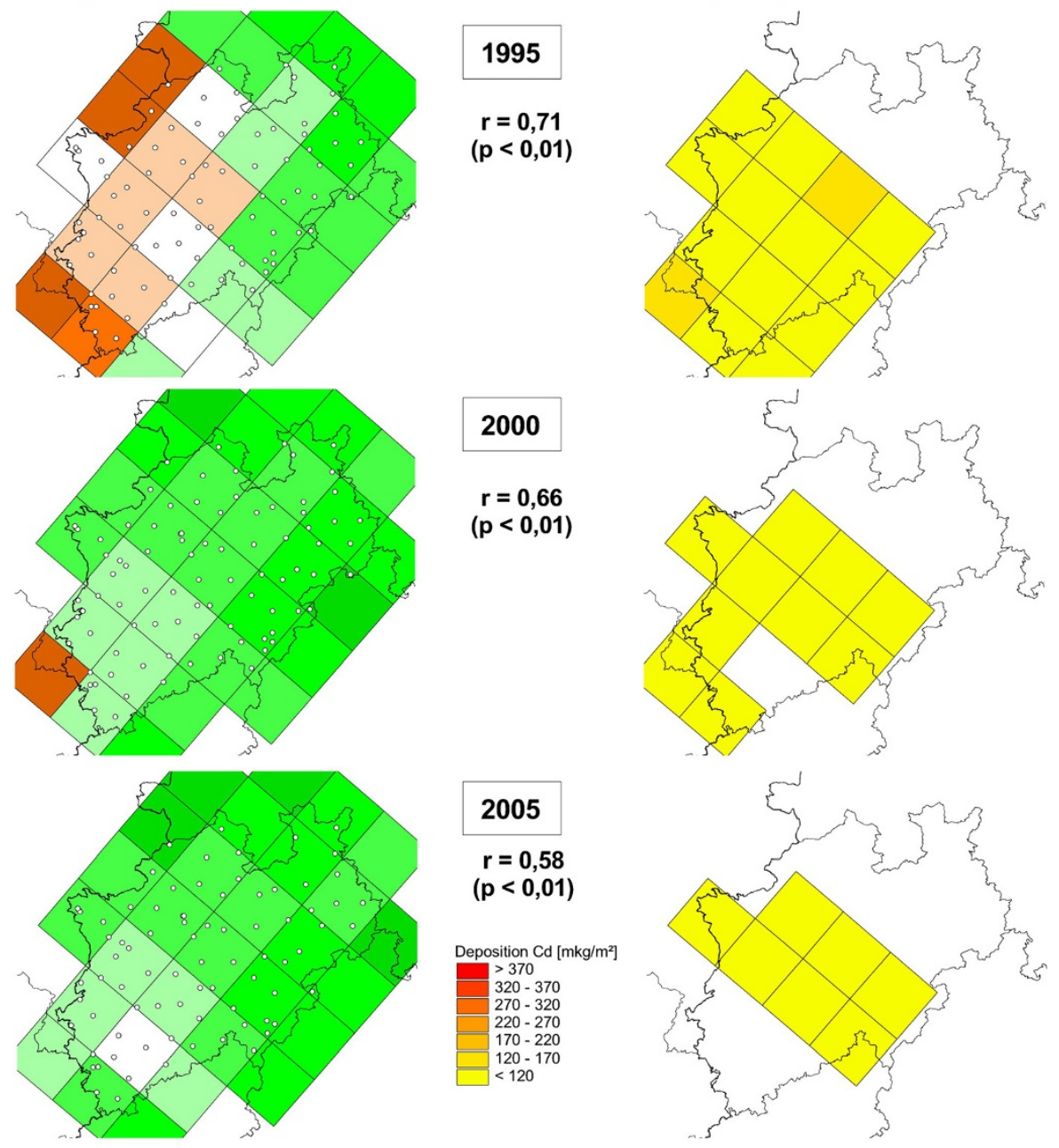

Abbildung 4 Vergleich Cd im Moos und in der modellierten Gesamtdeposition (EMEP).

unterfüttert werden: Bulk-Depositionsdaten stehen im Bundesgebiet für Freiland und Bestand im Falle von $\mathrm{Cd}$ von $\mathrm{n}=18$ Standorten zur Verfügung. Wet onlyDepositionsdaten (Freiland) stehen für Cd von sechs Standorten des UBA Luftmessnetzes und des ICP Integrated Monitoring zur Verfügung [22]. Die Rangkorrelationen zwischen den Metallkonzentrationen in Moosen einerseits und diesen Depositionsmessdaten sowie der von [34] modellierten Nass-, Trocken- und Gesamtdeposition andererseits variieren nach Stoffen und Depositionsmechanismus: Die statistischen Beziehungen zwischen den Konzentrationen in Bestandesdepositionen (bulk) und Moosen sind bei Cd ge-ring, im Falle der Freilanddeposition (bulk) mittel und bei der wet only-Deposition sehr hoch [22]. Die 


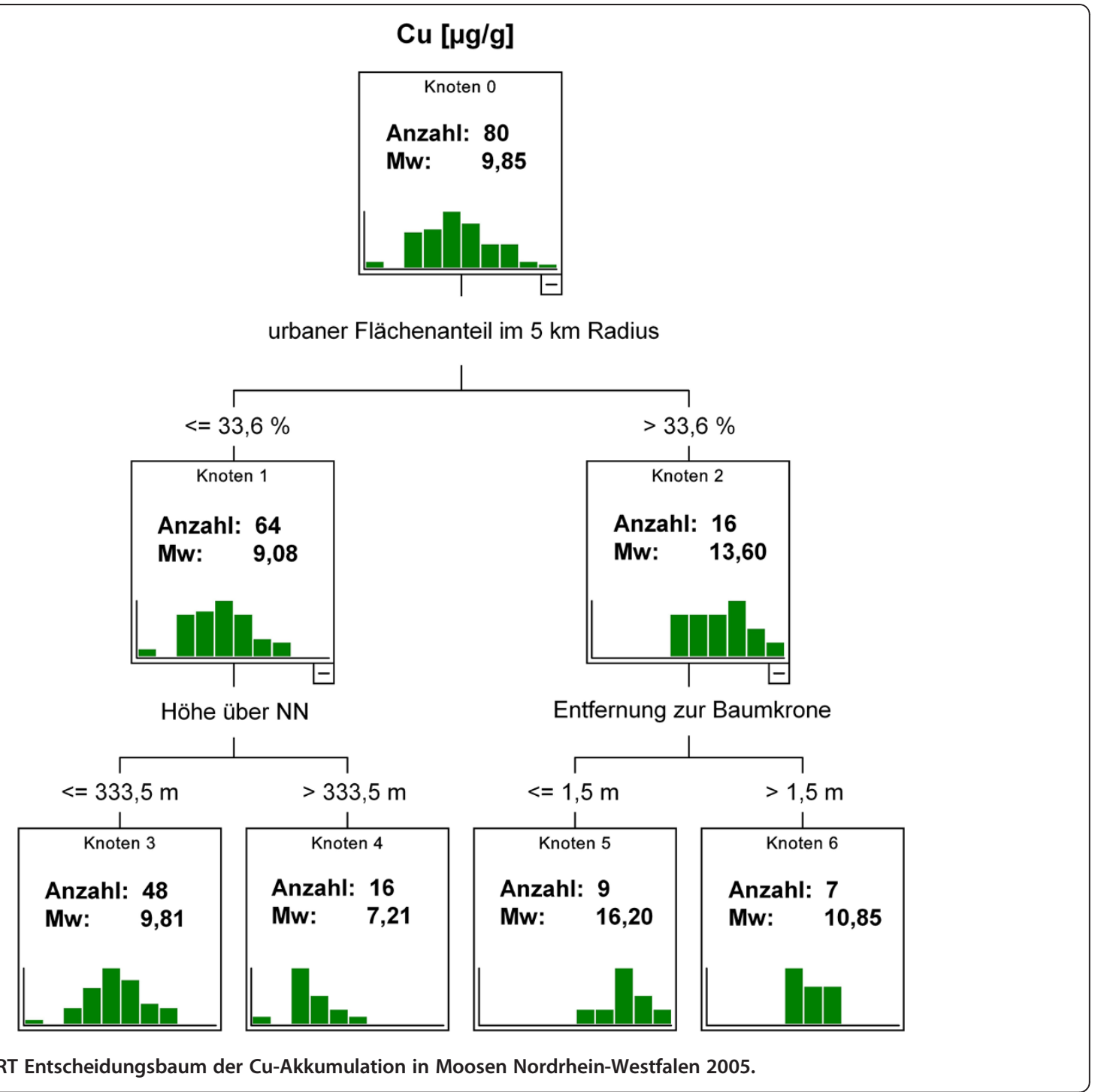

Korrelationen von Cd in Moosen und den von [34] modellierten Nass-, Trocken- und Gesamt-Depositionen sind niedrig bis mittel und variieren zeitlich, stoffspezifisch und nach modelliertem Depositionsprozess [22]. Auf europäischer Ebene lassen sich dies Befunde ebenfalls bestätigen: Cd-Konzentrationen der modellierten Gesamtdeposition (EMEP) und in Moosen sind mit $r_{s}=0.65$ korreliert [35].

Der Zeittrend der Metallbioakkumulation in NordrheinWestfalen unterscheidet sich von dem im Bundesgebiet und beispielsweise demjenigen in Baden-Württemberg: Von 2000 bis 2005 stiegen die die Konzentrationen fast aller Metalle in Nordrhein-Westfalen an, wobei die Zunahme bei $\mathrm{Cr}$ und $\mathrm{Zn}$ statistisch signifikant war. Die Stickstoffgehalte der Moose sind sowohl im innerdeutschen als auch im internationalen Vergleich hoch. Sie sind wie andernorts in Deutschland und Europa positiv mit dem Agrarflächenanteil im Umkreis der Beprobungsstellen verknüpft. Deutlicher als im Bundesdurchschnitt und einigen Bundesländern sind neben Moosart und -bewuchs, Waldflächenanteil, Flächendichte agrarischer Landnutzung, Niederschlagssumme, orografische Höhe, Bestandeshöhe und Entfernung der Moos-Entnahmestelle von Baumkronen oder Sträuchern auch der Flächenanteil urbaner Landnutzung sowie die Entfernung der Moossammelstellen zu Industrieanlagen mit den Stoffgehalten in den Moosen statistisch verknüpft.

\section{Schlussfolgerungen}

Die Agenda 21 verfolgt ebenso wie Nachhaltigkeitsstrategien von UNESCO MaB oder UNECE räumlich differenzierte Ansätze. Hierzu gehören auch die Instrumente, mit 
denen Zustand und Entwicklung der Umweltqualität erfasst werden: Umweltmonitoring soll für verschiedene Ebenen naturräumlicher und administrativer Raumgliederungen räumlich differenzierte und zuverlässige Daten aus langfristig betriebenen Messnetzen liefern. Das Moos-Monitoring ist ein Langfrist-Messnetz des UNECE ICP Vegetation. Anders als Depositionsmessnetze liefert das MoosMonitoring räumlich hoch aufgelöste Daten, die für die räumliche Modellierung der Depositionen und die räumlich differenzierte Erfassung und Bewertung von Stoffanreicherungen in der Umwelt unentbehrlich sind. Im Gegensatz zu Depositionsmessungen gibt das Moos-Monitoring Auskunft darüber, welche Stoffe und in welcher Menge an ausgewählten Rezeptoren der belebten Umwelt (Moos) ankommen. Erkenntnisse über solche Stoffanreicherungen sind für die Bewertung von Stoffeinträgen z.B. in Schutzgebieten aussagekräftiger als solche über atmosphärische Depositionen. Das MoosMonitoring liefert also nicht nur die Bestimmung der Umweltkonzentration von Stoffen, sondern immissionsschutzrechtlich relevante Beiträge zur Wirkungsermittlung, da die Anreicherung von Stoffen in Organismen die Vorstufe einer potenziellen physiologischen Wirkung bildet. Deshalb ist das MoosMonitoring insbesondere für Vorsorgemaßnahmen wichtig.

Das Moos-Monitoring sollte als Langfrist-Monitoring fortgeführt und zur Erfassung und Bewertung des Eintrags und der Anreicherung von Stoffen in Schutzgebieten genutzt werden. Um es noch stärker in Bezug zu den Inhalten von Nachhaltigkeitsstrategien zu setzen, sollte es enger mit dem Human-Biomonitoring (HBM) verknüpft werden und Beiträge $\mathrm{zu}$ dem Programm Transport Health and Environment Pan European Programme (THE PEP) von UNECE und WHO-Europe liefern (http://www.thepep.org/en/welcome.htm). Im HBM fehlt insbesondere die räumlich differenzierte Erfassung der Beziehungen zwischen der inneren Exposition von Probanden durch Human-Biomonitoring einerseits sowie der äußeren Exposition innerhalb und außerhalb von Gebäuden sowie in anthropogen wenig beeinflussten Naturräumen durch Umweltmonitoring andererseits [36,37].

\section{Interessenskonflikte}

Die Autoren erklären, dass keine Interessenkonflikte bestehen.

\section{Beiträge der Autoren}

Winfried Schröder leitete die Auswertungsstudie und schrieb den Text. Roland Pesch und Marcel Holy führten die statistische Datenauswertung und die GIS-Arbeiten durch. Lutz Genßler war verantwortlich für die empirischen Feldarbeiten und die Interpretation der Daten. Ferner ist Herr Genßler Mitglied im Arbeitskreis Bioindikation / Wirkungsermittlung, der die Durchführung der nationalen Moos-Monitoring-Kampagnen 1990 bis 2005 wissenschaftlich begleitete. Als Koordinator der europäischen MoosMonitoring-Kampagnen leistete Harry Harmens fachlichen Input aus Sicht des ICP Vegetation. Ilia llyin stellte die Ergebnisse der
Depositionsmodellierungen aus den EMEP-Programm für die vorliegende Untersuchung zur Verfügung.

\section{Author details}

'Lehrstuhl für Landschaftsökologie, Universität Vechta, Postfach 1553, 49364 Vechta, Germany. ${ }^{2}$ Obmann Arbeitskreis Bioindikation / Wirkungsermittlung der Landesanstalten und -ämter, Landesamt für Natur, Umwelt und Verbraucherschutz NRW, Leibnizstr. 10, Recklinghausen 45659, Germany.

${ }^{3}$ Chair of the International Cooperative Programme on Effects of Air Pollution on Natural Vegetation and Crops - ICP Vegetation, Centre for Ecology and Hydrology, Environment Centre Wales, Deiniol Road, Bangor, Gwynedd LL57 2UW, UK. ${ }^{4}$ Meteorological Synthesizing Centre East of EMEP, Krasina pereulok, 16/1, Moscow 123056, Russia.

Received: 10 June 2011 Accepted: 23 December 2011 Published: 15 March 2012

\section{References}

1. Harmens H, Norris D and the participants of the moss survey: Spatial and temporal trends in heavy metal accumulation in mosses in Europe (19902005). Programme Coordination Centre for the ICP Vegetation, Centre for Ecology and Hydrology. Bangor, UK: Environment Centre Wales; 2008.

2. Bealey W, Cape JN, Leith ID, Long S, Kinnerlsey RP: Air quality outcomes in pollution regulation: strengths, limitations and potential. Science Report SC030175/SR1, CEH Project Number: C02600. Bristol: Environment Agency; 2008:1-47.

3. Bealey WJ, Long S, Spurgeon DJ, Leith I, Cape JN: Review and implementation study of biomonitoring for assessment of air quality outcomes. Science Report - SC030175/SR2. Bristol: Environment Agency; 2008:1-170.

4. Forster E-M, Matthies M, Brüggemann R: Optimierung eines BioindikatorMeßnetzes mit geostatistischen Methoden und einem geographischen Informationssystem. Umweltwiss Schadst Forsch 1993, 5:286-294.

5. Genßler L, Rademacher J, Rammert U: Arbeitskreis der Landesanstalten und -ämter Konzeption der künftigen Aufgabenbereiche. Umweltwiss Schadst Forsch 2001, 13:1-4.

6. Herpin U, Siewers U, Markert B, Rosolen V, Breulmann G, Bernoux M: Second German heavy-metal survey by means of mosses, and comparison of the first and second approach in Germany and other European countries. Env SC Pollut Res 2004, 11:57-66.

7. Kostka-Rick R, Leffler US, Markert B, Herpin U, Lusche M, Lehrke J: Biomonitoring zur wirkungsbezogenen Ermittlung der Schadstoffbelastung in terrestrischen Ökosystemen Konzeption, Durchführung und Beurteilungsmaßstäbe im Rahmen von Genehmigungsverfahren. Umweltwiss Schadst Forsch 2001, 12:5-12.

8. Mohr K: Passives Monitoring von Stickstoffeinträgen in Kiefernforsten mit dem Rotstengelmoos (Pleurozium schreberi (Brid) Mitt). Umweltwiss Schadst Forsch 1999, 11:267-274.

9. Mohr K: Biomonitoring von Stickstoffimmissionen. Möglichkeiten und Grenzen von Bioindikationsverfahren. Umweltwiss Schadst Forsch 2007, 19:255-264.

10. Rühling A, Tyler G: An ecological approach to the lead problem. Botaniska Notiser 1968, 121:321-343.

11. Rühling $A$, Tyler $G$ : Ecology of heavy metals - a regional and historical study. Botaniska Notiser 1969, 121:248-259.

12. Rühling A, Tyler G: Sorption and retention of heavy metals in the woodland moss Hylocomium splendens (Hedw.) Br. Et Sch. Oikos 1970, 21:248-342.

13. Schröder W, Englert C, Pesch R, Zechmeister HG, Thöni L, Suchara I, Maňkovská B, Jeran Z, Harmens H, Grodzinska K, Alber R: Metallakkumulation in Moosen: Standörtliche und regionale Randbedingungen des Biomonitoring von Luftverunreinigungen. Umweltwiss Schadst Forsch 2008, 20:120-132.

14. Tyler G: Bryophytes and heavy metals: a literature review. Bot I Linnean Soc 1990, 104:231-253.

15. Wappelhorst O, Korhammer S, Leffler US, Markert B: Ein Moosbiomonitoring zur Ermittlung atmosphärischer Elementeinträge in die Euroregion Neiße (D, PL, CZ). Umweltwiss Schadst Forsch 2000, 12:191-200.

16. Zechmeister HG, Dullinger S, Hohenwallner D, Riss A, Hanus-Illnar A, Scharf $\mathrm{S}$ : Pilot study on road traffic emissions (PAHs, heavy metals) measured by using mosses in a tunnel experiment in Vienna, Austria. Env SC Pollut Res 2006, 13:398-405. 
17. Ilyin I: Modelled EMEP heavy metal deposition vs moss measurements: Evaluation of spatial patterns and log-term temporal trends. In Proceedings of the 22nd Task Force Meeting of the ICP Vegetation, 2 - 5 February 2009; Braunschweig. Edited by Harmens H, Mills G, Menichino N, Bender J, Weigel H. Bangor, UK: ICP Vegetation Programme Coordination Centre, Centre for Ecology and Hydrology; 2009:27.

18. Ilyin I, Travnikov O, Aas W: Heavy metals: transboundary pollution of the environment. EMEP/MSC-E Status Report 2/2006. Moscow, Russian Federation: Meteorological Synthesizing Centre - East; 2006. http://www.msceast.org.

19. Harmens $\mathrm{H}$ : Monitoring of atmospheric deposition in Europe using bryophytes. Monitoring manual 2005/2006 survey. Bangor, UK: ICP Vegetation Programme Coordination Centre, Centre for Ecology and Hydrology; 2005.

20. Steinnes E, Rühling $\AA$, Lippo H, Mäkinen A: Reference materials for largescale metal deposition surveys. Accredit Qual Assur 1997, 2:243-249.

21. Funk W, Dammann V, Donnevert G: Quality assurance in analytical chemistry. Applications in environmental, food and materials analysis, biotechnology and medical engineering. 2nd edition. Weinheim: Wiley-VCH; 2006.

22. Schröder W, Pesch R, Matter $Y$, Göritz A, Genßler L, Dieffenbach-Fries H: Trend der Schwermetall-Bioakkumulation 1990 bis 2005: Qualitätssicherung bei Probenahme, Analytik, geostatistischer Auswertung. Umweltwiss Schadst Forsch 2009, 21:549-574.

23. Pesch R, Schröder W, Genßler L, Göritz A, Holy M, Kleppin L, Matter Y: MoosMonitoring 2005 / 2006: Schwermetalle IV und Gesamtstickstoff. Berlin: Umweltforschungsplan des Bundesministers für Umwelt, Naturschutz und Reaktorsicherheit. FuE-Vorhaben 20564 200, Abschlussbericht, im Auftrag des Umweltbundesamtes, 90 S, 11 Tab, 2 Abb. (Texteil); 51 S. + 41 Karten, 34 Tabellen, 46 Diagramme (Anhangsteil); 2007.

24. Pesch R: Geostatistische und multivariat-statistische Analyse des MoosMonitorings 1990, 1995 und 2000 zur Ableitung von Indikatoren für die Bioakkumulation atmosphärischer Metalleinträge in Deutschland. Dissertation, Hochschule Vechta: Lehrstuhl für Landschaftsökologie; 2003.

25. Matheron G: Les variables régionalisées et leur estimation. Paris: Masson; 1965.

26. Webster R, Oliver MA: Geostatistics for environmental scientists. Chichester, New York, Weinheim, Brisbane, Singapore, Toronto: Wiley; 2001.

27. Johnston K, Ver Hoef JM, Krivoruchko K, Lucas N: Using ArcGIS Geostatistical Analyst. Redlands; 2001

28. Kleppin L, Schröder W, Pesch R, Schmidt G: Entwicklung und Erprobung einer Metadaten- und WebGIS-Applikation für das Expositionsmonitoring mit Moosen in Deutschland Ein Beitrag zum LTER-Netzwerk. Umweltwiss Schadst Forsch 2008, 20:38-48.

29. Pesch R, Schmidt G, Schröder W, Aden C, Kleppin L, Holy M: Development, implementation and application of the Web-GIS MossMet. In The geospatial web. How geo-browsers, social software and the Web 2.0 are shaping the network society. Edited by Tochtermann K, Scharl A. London: Springer; 2007:191-200.

30. Keil M, Kiefl R, Strunz G: CORINE Land Cover 2000 - Germany. Final Report. German Aerospace Center. Oberpfaffenhofen: German Remote Sensing Data Center; 2005.

31. Breimann L: Friedmann J A, Olshen R A, Stone C J: Classification and regression trees. Belmont, CA: Wadsworth; 1984.

32. Dämmgen U: Bestimmung von Ammoniak-Einträgen aus der Luft und deren Wirkungen auf Waldökosysteme. Braunschweig: ANSWER-Projekt, Landbauforschung Völkenrode. Sonderheft 279; 2005:1-113.

33. Siewers U, Herpin U, Straßburger S: Schwermetalleinträge in Deutschland Moos-Monitoring 1995. Teil 2. Geologisches Jahrbuch, Sonderhefte, Heft SD 3. Stuttgart: Bornträger; 2000.

34. Gauger T, Anshelm F, Schuster H, Erisman JW, Vermeulen AT, Draaijers GPJ, Bleeker A, Nagel HD: Mapping of ecosystem specific long-term trends in deposition loads and concentrations of air pollutants in Germany and their comparison with Critical Loads and Levels: Part 1: Deposition Loads 19901999. Berlin: Final Report 29942210 on behalf of Federal Environmental Agency (Umweltbundesamt); 2002.

35. Holy M, Schröder W, Pesch R, Harmens H, Ilyin I, Steinnes E, Alber R, Aleksiayenak Y, Blum O, Coskun M, Dam M, de Temmermann L, Frolova M, Frontasyeva M, Gonzalez Miqueo L, Grodzińska K, Jeran Z, Korzekwa S, Krmar M, Kubin E, Kvietkus K, Leblond S, Liiv S, Magnússon S, Maňkovská B, Miodrag K, Piispanen J, Rühling A, Santamaria J, Spiric Z, Suchara I, Thöni L, Urumov $V$, Yurukova $L$, Zechmeister: First thorough identification of factors associated with $\mathrm{Cd}, \mathrm{Hg}$ and $\mathrm{Pb}$ concentrations in mosses sampled in the European Surveys 1990, 1995, 2000, and 2005. J Atmosph Chem 2010, 63:109-124.

36. Pesch R, Schmidt G, Schröder W, Conrad A, Kolossa-Gehring M, Feigenspan S, Utermann J: Das Potenzial raumbezogener Daten im HumanBiomonitoring am Beispiel des Kinder-Umwelt-Surveys. In Angewandte Geoinformatik 2009. Edited by Strobl J, Blaschke T, Griesebner G. Heidelberg: Wichmann; 2009:248-257.

37. Zechmeister HG, Köllensperger G, Hann S, Hanus-IInar A, Scharf S, Schneider J, Neuberger M, Moshammer H, Hohenwallner D, Schierl R, Schröder W, Schmidt G, Pirintsos SA, Loppi S, Gaggi C, Nante N, Namiesnik J, Zygmunt B, Jeran Z, Perharic L, Künzli N, Frontasyeva M, Lyapunov S, Ilchenko I, Harmens H: INDOMO. New tool for monitoring indoor pollutants with health relevance across Europe. Wien; 2007.

doi:10.1186/2190-4715-24-12

Cite this article as: Schröder et al: Akkumulation von Metallen und Stickstoff in Moosen in Nordrhein-Westfalen 1990 - 2005 (Accumulation of metals and nitrogen in mosses in North Rhine-Westfalia 1990-2005). Environmental Sciences Europe 2012 24:12.

\section{Submit your manuscript to a SpringerOpen ${ }^{\odot}$ journal and benefit from:}

- Convenient online submission

- Rigorous peer review

- Immediate publication on acceptance

- Open access: articles freely available online

- High visibility within the field

- Retaining the copyright to your article

Submit your next manuscript at $>$ springeropen.com 